
2 Research Square
Preprints are preliminary reports that have not undergone peer review.
They should not be considered conclusive, used to inform clinical practice, or referenced by the media as validated information.

\title{
The Embryo Characteristics and Genes Expression Analysis Involved in Apomixis of Wild Germplasm Materials of Kentucky Bluegrass in Gansu Province of China
}

\author{
Jinqing Zhang \\ Gansu Agricultural University \\ Huiling Ma ( $\square$ mahl@gsau.edu.cn ) \\ Gansu Agricultural University
}

\author{
Research Article \\ Keywords: Apomixis, Poa pratensis, Transcriptome analysis, Sexual reproduction, Meiosis \\ Posted Date: January 18th, 2021 \\ DOI: https://doi.org/10.21203/rs.3.rs-146413/v1 \\ License: (c) (i) This work is licensed under a Creative Commons Attribution 4.0 International License. Read Full License
}




\section{Abstract}

Background: Apomixis is mainly used to maintain the heterosis, stability, and consistency of crops. Its main advantage is to reduce the costs of seed production and shorten the breeding process. In the field of hybrid breeding, apomixis has been referred to as the "asexual revolution" and has realized a new green revolution.Apomixis is mainly used to maintain the heterosis, stability, and consistency of crops. Its main advantage is to reduce the costs of seed production and shorten the breeding process. In the field of hybrid breeding, apomixis has been referred to as the "asexual revolution" and has realized a new green revolution. Kentucky bluegrass (Poa pratensis) is a natural apomictic species that mainly exhibits facultative apospory. Its main feature is that the somatic nucellar cells bypass meiosis and double fertilization to form unreduced embryos, and the development of the endosperm requires pseudogamy. Although apomixis is of great significance in breeding, the genetic control of apomixis remains unclear. Therefore, we report the results of a global gene expression analysis of wild germplasm materials of Kentucky bluegrass spikelets in Gansu province of China, exhibiting significant differences in apomictic rates to identify the genes, biological processes, and molecular functions related to apomixis.

Result: At four reproductive periods, there were 5400 differentially expressed genes (DEGs) between the two genotypes, including 2476 downregulated and 2823 upregulated genes. Further analysis of the gene functions, pathways, expression patterns, networks, and transcription factors (TFs) showed that the occurrence of apomixis in Kentucky bluegrass was related to changes in the time- and space-related expression of genes associated with sexual reproduction, which led to disordered sexual reproduction and thus the production of offspring by apomixis.

Conclusion: At the transcriptional level, the genesis and development of apomixis was regulated by TFs. It also involved the coexpression of many genes associated with disordered meiosis, hormone signal transduction, embryonic development, stress response pathways, and epigenetics. We also verified these 16 DEGs by real-time quantitative PCR. The expression results were basically consistent with the transcriptome expression profile, indicating that the transcriptome data were reliable. The results of this study may provide a theoretical basis for revealing the mechanism of occurrence and development of apomixis in Kentucky bluegrass.

\section{Background}

Kentucky bluegrass (Poa pratensis) is mainly distributed in cold and humid regions in the cold and northern temperate zones[1, 2]. It is not only an excellent forage grass in many natural grasslands[3], but also widely used in lawn establishment[4]. Kentucky bluegrass can also be used to stabilize soil erosion and improve soil structure and fertility[5]. In addition, there is research interest in Kentucky bluegrass not only for its turf and feed practicability, but also for its remarkable evolutionary history and adaptability. Thousands of years of infiltration and natural selection have resulted in Kentucky bluegrass evolving into one of the most volatile, buffering, and tolerant species. Genomic plasticity and reproductive diversity are two interrelated characteristics that are considered to be integral to its success[6]. As early as 1933, Müntzing[7] reported that Kentucky bluegrass was mainly aposporic, following which it was discovered that it possesses a facultative apomictic system in which apospory and sexual hybridization or self-crossing coexist[8, 9]. Furthermore, the apomictic rate varies greatly depending on the variety and source, ranging from 0 to 100\%[10]. Therefore, Kentucky bluegrass can be used as a "model" species for the study of plant apomixis and its inheritance[11, 12]. The apospory process of Kentucky bluegrass involves the specialization of one or more nucellus cells in the ovary to form aposporous initial cells (AICs), which further differentiate, expand, and undergo mitosis to form unreduced embryo sacs. They do not need to be fertilized to form apomictic embryos[13]. When there is pseudogamy, these apomictic embryos will develop into active seeds through parthenogenesis[14].

Apomixis is a special process of reproduction through seeds, acquiring the ability to bypass sexual reproduction (meiosis and fertilization). It involves a combination of three basic events: 1) apomixis, which involves the loss or failure of meiosis; 2) parthenogenesis, whereby embryogenesis is independent of fertilization, and 3) pseudogamy, involving functional endosperm formation after autonomic or fertilization[15]. Apomixis does not require gene recombination and alignment[16], and thus it can fix heterosis[17, 18] and avoid the incompatibility obstacle of sexual reproduction and the transfer of viruses in asexual plants[19]. In addition, apomixis has great potential in improving breeding efficiency and reducing the cost of seed production[20]. In the field of hybrid breeding, it has been referred to as the "asexual reproduction revolution", achieving a new green revolution[21]. In addition, facultative apomixis has greater versatility, adaptability, and evolutionary ability compared to obligate reproduction[22]. At present, the research on apomixis has mainly involved the identification of its related genes. There are two commonly used methods: 1) screening and analysis of sexual model plant mutants showing asexual components; and 2) identification of candidate genes that are genetically and/or functionally related to apomixis in natural apomictic species. These two methods are closely related and have made significant contributions to apomixis research[23]. For example, some candidate genes related to apomixis have been found, such as SERK[24, 25], LEC[26], PGA6/WUS[27], and SWI7[28]. However, although apomixis has been described in more than 400 angiosperms[29], there are no natural apomictic species in major seed crops, and attempts to introduce apomictic characteristics into crops through conventional breeding methods have largely been unsuccessful[30]. Although apomixis is of great significance in crop breeding, and a large number of studies have been conducted, our current understanding of the molecular mechanisms of its development is still largely unknown[16, 23]. In recent years, the high-throughput sequencing technology of cDNA fragment population (RNA-seq) has developed rapidly and has become the most effective way to predict new transcripts and identify differentially expressed genes (DEGs) in different tissues, genotypes, abiotic conditions, and developmental stages at the same time. It can be used for the rapid analysis and in-depth study of the transcriptome in any species. Compared with traditional microarray analysis, which is often used in gene expression research, RNA-seq technology does not require probes specific to species or transcripts, nor does it require cloning libraries or any prior knowledge about the genomes of species[16]. In addition, RNA-seq technology can also detect a higher proportion of DEGs, especially those with low levels of expression.

Wieners et al.[9] pointed out that to improve Kentucky bluegrass, such as by reducing its management investment its production, improving its resistance to diseases and insect pests, resisting low pruning, or improving other important agronomic traits, it is necessary to collect and identify different germplasms. Gansu, China, which is located at the intersection of the Qinghai-Tibet Plateau, Loess Plateau, Mengxin Plateau, and West Qinling Mountains, has complex topography and climate and is rich in Kentucky bluegrass resources. There are six endemic Kentucky bluegrass species in Gansu alone, making the collection of different wild germplasm materials of Kentucky bluegrass convenient[31]. Although Kentucky bluegrass is closely related to crops such as wheat (Triticum

Page 2/24 
aestivum), rice (Oryza sativa), and maize (Zea mays), its apomictic trait cannot easily be transformed into cereal crops by hybridization, and there is a lack of genomic information available for Kentucky bluegrass. In this case, it is crucial to analyze its gene expression patterns during embryonic development. Therefore, in this study, we selected wild Kentucky bluegrass germplasm resources in Gansu exhibiting significant differences in apomictic rates and used high-throughput sequencing to obtain spikelet reference transcriptomes of two genotypes of Kentucky bluegrass, screen and identify relevant DEGs, and clarify the biological processes that these genes may participate in. Our findings should help elucidate the molecular basis of apomixis in Kentucky bluegrass and provide basic data for the cultivation of excellent new varieties of Kentucky bluegrass through apomixis.

\section{Plant Materials And Methods}

\section{Experiment material}

We collected wild germplasm materials of Kentucky bluegrass from various areas in Gansu, China, in the early stage, and the apomictic rate of these wild germplasm materials was calculated using cytoembryology, as described in our previous study[80]. In this study, wild germplasm materials of Kentucky bluegrass collected from Longnan (LN) and Gannan (GN), which exhibited significant differences in apomictic rates, were selected as research materials. Information on the collection sites is shown in Table 4. In late April 2019, GN and LN were planted in the lawn training base of Gansu Agricultural University (longitude $103^{\circ} 34^{\prime}$ east, latitude $36^{\circ} 5^{\prime}$ north). The climatic conditions and soil information of the lawn training base are shown in Table 5 . The spikelets of Kentucky bluegrass in the premeiosis (PreM), meiosis (M), postmeiosis (PostM), and anthesis (A) stages were collected according to the panicle type reported by Albertini et al.[25]. Some of the spikelets were immediately fixed in FAA (formalin: glacial acetic acid: $70 \%$ ethanol $=1: 1: 18$ ) and then used to make paraffin sections. Some of the spikelets were rapidly frozen in liquid nitrogen and stored at $-80^{\circ} \mathrm{C}$ for endogenous hormone determination and RNA extraction. Three biological replicates were set at each time point, and each sample was taken from a mixed sample of at least five plants.

\section{Cytoembryological observation of the ovaries of Kentucky bluegrass}

After the florets of the spikelets stored in $70 \%$ ethanol were separated, the samples were subjected to an ethanol dehydration series. Xylene was applied as a transparency agent, the samples were dipped in paraffin wax, and a split paraffin embedding machine (EG1150H+EG1150C) was then used for embedding. A Leica RM2265 electric rotary automatic microtome was used to produce sections of $10 \mu \mathrm{m}$ thickness. Ehrlich (Ehrlich's) hematoxylin (1 min)-eosin (2 min) double staining was conducted in a Leica RM2265 automatic dyeing machine, following which a Revolve RVL-100-G inverted microscope was used to observe and photograph the samples.

\section{Determination of endogenous hormones in the spikelets of Kentucky bluegrass}

The endogenous hormones of wild Kentucky bluegrass were determined by high-performance liquid chromatography (HPLC). Indole-3-acetic acid (IAA), gibberellin (gibberellin, $\mathrm{GA}_{3}$ ), abscisic acid (ABA), and zeatin (ZT) were tested. SPSS 19.0 (IBM Corp., Armonk, NY, USA) was used for independent samples $t$ tests, and Microsoft Excel 2016 (Microsoft Corp., Albuquerque, NM, USA) was used for producing graphs.

\section{RNA extraction, library construction, and Illumina sequencing}

Total RNA was extracted from all samples using an RNasy Plant Mini Kit according to the manufacturer's instructions (Qiagen, Hilden, Germany). After accurate detection of RNA by $1 \%$ agarose gel electrophoresis as well as a NanoPhotometer spectrophotometer (IMPLEN, Westlake Village, CA, USA) and Agilent 2100 biological analyzer (Agilent Technologies, Palo Alto, CA, USA), the cDNA library was constructed using Illumina's NEBNext ${ }^{8}$ UltraTM RNA Library Prep Kit (San Diego, CA). First, polyA mRNA was screened and enriched by Oligo (DT) magnetic beads. The mRNA was then randomly interrupted in NEB Fragmentation Buffer and used as a template, and random oligonucleotides were used as primers to synthesize the first-strand cDNA in a M-MuLV reverse transcriptase system. Third, the RNA chain was degraded by RNaseH, and the second chain of cDNA was synthesized from dNTPs in the system of DNA polymerase I. Finally, cDNA of 250-300 bp was obtained and subjected to end-repair and polyA tail addition and screened using AMPure XP beads. PCR amplification and purification of the PCR products were carried out, and the final library was obtained.

The constructed library was sequenced on an Illumina HiSeq 4000 platform ( $2 \times 150$ bp reading length) after passing a quality control test. Raw reads were obtained by sequencing and were filtered to obtain clean reads. The transcripts of the clean reads were assembled by Trinity[81] software, and the longest among similar classes was selected as the unique gene by CD-HIT[82], and a collection of all gene sequences was obtained as a reference sequence for analyzing gene expression. Finally, RSEM software[83] was used for reference sequence mapping of the clean reads.

\section{Functional annotation of unigenes}

BLASTX was used to search unigenes in the Nr (NCBI non-redundant protein sequences), Nt (NCBI nucleotide sequences), Swiss-Prot, and KOG (euKaryotic Ortholog Groups) databases, and the Pfam (Protein family) database was used to predict protein domains.

The expression level of each transcript was determined by FPKM (the number of fragments per thousand exons per million mapped fragments). The DESeq R Bioconductor package[84] was used to determine the differential expression of expressed genes based on the negative binomial distribution model. Benjamini and Hochberg[85] was used to control the false discovery rate (FDR). A $\mid \log 2$ (FoldChange) $\mid \geq 1$ and FDR $<0.05$ were used as the criteria for selecting DEGs. When the Bonferroni-corrected $P$-value was $<0.05$, a DEG was considered to be significantly enriched. Using the method of GOseq[86], unigenes were annotated according to biological process, cellular composition, and molecular function in the GO (Gene Ontology) database, so as to obtain the biological function of significantly enriched DEGs. KEGG (Kyoto Encyclopedia of Genes and Genomes) and related software KAAS (KEGG Automatic Annotation Server, Tokyo, Japan)[87] were used to analyze the metabolic pathways. 


\section{Quantitative real-time (qRT) PCR verification}

Sixteen genes were randomly selected and specific PCR primers were designed by using the NCBI online website Primer-BLAST. The total RNA was extracted using an RNeasy Plant Mini Kit according to the manufacturer's instructions (Qiagen, Hilden, Germany). The total RNA was reverse-transcribed into cDNA using a Prime Script RT reagent Kit with a gDNA Eraser (Perfect Real Time) (TaKaRa, Japan) and diluted 20 times as a template. The qRT-PCR was performed with a StepOnePlus ${ }^{\text {TM }}$ Real-Time PCR System (ABI) using SYBR premix Ex Taq (Takara, Japan). The reaction volume was $20 \mu \mathrm{L}$ and included $2 \times$ SuperReal PreMix Plus $10 \mu \mathrm{L}, \mathrm{ddH}_{2} \mathrm{O} 3 \mu \mathrm{L}, \mathrm{cDNA}$ template $5 \mu \mathrm{L}$, and forward and reverse primer $1 \mu \mathrm{L}$. The reaction conditions included pre-denaturation at $95^{\circ} \mathrm{C}$ for 15 min and 40 cycles of PCR amplification, including denaturation at $95^{\circ} \mathrm{C}$ for $10 \mathrm{~s}$ and annealing at $58^{\circ} \mathrm{C}$ for $30 \mathrm{~s}$. Using Actin as the internal reference gene, the relative expression level of the selected gene was calculated by $2^{-\Delta \Delta C t}[88]$.

\section{Results}

\section{Histological study on the ovaries of Kentucky bluegrass}

The sexual embryo sac of Kentucky bluegrass is a typical polygonum-type embryo sac, and the normal sexual reproductive embryo sac develops from the MMC through meiosis (Fig. 1A-G). The MMC develops from a cell located at the top of the central axis cell mass under the nucellus epidermis. Compared with other nucellar cells, this cell is large in size and has a small and dense cytoplasm, and the nucleus accounts for the main part of the whole cell, which is called an archesporial cell (Fig. 1A). The archesporial cell grows and develops into MMC (Fig. 1B). Then the MMC undergoes meiosis to form a dyad and tetrad (Fig. 1C and D). With growth and development, the tetrad forms a megasporangium (Fig. 1E). The megasporangium undergoes mitosis gradually to form a two-nucleus, four-nucleus embryo sac and a "7-cell-8-nucleus" structure (Fig. 1F and G). At this point, the development of the female sexual gametophyte of Kentucky bluegrass is complete.

Developmental variety in the AICs was observed (Fig. 1H-P). First, the multiple nucellar cells in the ovary were specialized, and the cell volume was significantly larger than that of the surrounding nucellar cells $(F i g .1 \mathrm{H})$. During the stage of the archesporial cell, the specialization of the nucellar cells also occurred (Fig. 1I). Subsequently, the specialized nucellar cell developed and expanded to form an AIC, which was large in size and had an obvious nucleus. The AIC could coexist with MMC, and they could be arranged horizontally (Fig. 1J) or longitudinally (Fig. 1K). In addition, multiple specialized nucellar cells developed together to form multiple AICs. At this time, the MMC may disintegrate and form nutrients that are absorbed by the AICs (Fig. $1 \mathrm{~L}$ ). In some ovaries, the MMC developed normally and coexisted with two AICs (Fig. 1M). The AIC could form and coexist after the MMC formed a dyad (Fig. 1N). The AIC formed a longitudinally arranged two-nucleus embryo sac that was clearly distinguished from the development of sexual reproduction after mitosis (Fig. 10). In addition, the existence of apomixis led to the formation of a three-nucleus embryo sac (Fig. 1P).

\section{Changes in endogenous hormone contents in the spikelets of Kentucky bluegrass}

The change trend in ZT content of the spikelets of the two genotypes was basically the same, increasing at first and then decreasing, and reaching the maximum value at meiosis. One difference was that the increase and decrease in GN materials were higher than that in LN (Fig. $2 A)$. The variation trend of $\mathrm{GA}_{3}$ content in both genotypes first increased and then decreased, being highest at meiosis in $\mathrm{LN}$ and highest at the postmeiosis stage in GN (Fig. $2 \mathrm{~B}$ ). The content of IAA in LN exhibited no difference between the first two periods and was significantly lower than that in the latter two periods. In GN, the content of IAA decreased first, then increased, and finally decreased. Comparing the two genotypes, the IAA content of LN was significantly lower than that of GN in the first two periods and was higher in the latter two periods (Fig. 2C). The ABA content increased gradually with the development of the spikelets in both genotypes, and there was no difference between the two genotypes in the first three periods. Only at anthesis was the ABA content in LN significantly higher than that in GN (Fig. 2D).

\section{RNA sequencing analysis}

Each sample had more than 21.03 million raw reads, and a total of $152.35 \mathrm{~Gb}$ clean reads were obtained after filtering. With the exception of LM, the error rate of the other samples was $0.02 \%$. The Q20 and Q30 percentages were greater than $98.12 \%$ and $94.46 \%$, respectively, and the GC content percentage ranged from $52.17 \%$ to $54.06 \%$ (Table 1). Furthermore, the clean reads of each sample were mapped to obtain the total mapped reads after filtering. The percentage of total mapped reads in all samples to total reads was more than $63.05 \%$ (Table S1). In addition, we found there were 833 (57.85\%) complete sequences, 366 (25.42\%) fragment sequences, and 241 (16.74\%) homologous genes (Fig. S1).

\section{Transcriptome de novo assembly and annotation}

A total of 892774 transcripts and 288678 unigenes were obtained. The length and quantity statistics of the transcripts and unigenes are shown in Fig. $3 \mathrm{~A}$. According to the statistics, there were 300685 and 114763 transcripts and unigene sequences with a length of 300 to 500 bp, respectively. The number of transcripts between 501 and 1000 bp was the largest, accounting for $40.90 \%$ of the total. The number of transcripts and unigenes larger than 2000 bp was the lowest, accounting for only $4.70 \%$ and $4.92 \%$ of the total, respectively (Fig. 3B, Table S2, Fig. S2). Furthermore, functional annotation information of the unigenes of the Kentucky bluegrass spikelet transcriptome was obtained (Fig. 3C, Table S3). The number of annotations in the Nr database was the largest, accounting for $47.51 \%$. The results showed that the spikelet gene sequence of Kentucky bluegrass had the highest homology with Aegilops tauschii, followed by Brachypodium distachyon and Hordeum vulgare. The homology rates of Triticum aestivum and Triticum urartu accounted for $7.3 \%$ and $7.2 \%$, respectively (Fig. 3D). In addition, analyzing the distribution of its e-value, it was found that only $1.2 \%$ of the unigenes annotated to the $\mathrm{Nr}$ database were compared to an e-value equal to 0 (Fig. S3A). The distribution of sequence similarity showed that $33.8 \%$ of the Nr database-annotated unigenes reached $80 \%-95 \%$ similarity, and only $15.2 \%$ of the sequence similarity was less than $60 \%$, which indicated that the sequence similarity of the species was relatively high (Fig. S3B). 


\section{Identification and analysis of DEGs}

In LN, the number of DEGs was the highest in LA vs. LPreM, which had 11849 upregulated and 19019 downregulated genes. The number of DEGs was lowest in LPostM vs. LM, which included 176 and 147 upregulated and downregulated genes, respectively (Fig. 4, Table 2). The change in GN was the same as that of LN, in which the number of DEGs was the largest in GA vs. GpreM and the lowest in GPostM vs. GM (Table 2, Table S4). To analyze the specific expression of genes of the four stages and visually illustrate the common and unique DEGs, we constructed a Venn diagram of the DEGs (Fig. 4). We further analyzed the DEGs between the two genotypes. It was found that 23234 DEGs were identified at PreM, of which 13583 were upregulated and 9651 were downregulated. A total of 37773 DEGs were identified in LM vs. GM, including 19507 upregulated and 18266 downregulated genes, respectively. The number of upregulated and downregulated genes identified was 22722 and 23446, respectively, at PostM. A total of 15691 and 9766 upregulated and downregulated DEGs were identified in LA vs. GA, respectively (Fig. 4C, Table S4). There were 5400 DEGs expressed at all four stages. The number in PreM was the lowest, only accounting for $8.76 \%$ of the total DEGs, while the percentage of DEGs in PostM was the highest (23.05\%).

\section{Functional annotation of DEGs}

We selected GO terms that were significantly enriched in at least one stage and that may be related to apomixis to construct a heatmap showing expression characteristics (Fig. 5). We found that the DEGs were generally related to stress response (regulation of response to stress, response to oxidative stress, and oxidoreductase activity), hormone signal (regulation of hormone levels, hormone metabolic process, cellular hormone metabolic process), meiotic (reciprocal meiotic recombination), female gamete generation (female gamete generation), and cell wall (cell wall modification) (Fig. 5, Table S5). Among them, only oxidoreductase activity was significantly enriched at all four stages, cell wall was significantly enriched at three stages, and the other 10 terms and 6 terms were only significantly enriched at two and one stages, respectively. To further explore the biological pathways of the DEGs, we performed KEGG analysis (Table S6). The results indicated that the pathways with the highest scores were photosynthesis-antenna proteins, thiamine metabolism, flavone and flavonol biosynthesis, and cutin, suberine, and wax biosynthesis. According to existing research reports, we listed the pathways that may be related to apomixis in this study (Table 3). Although the scores of these pathways are not the highest, they were still found.

\section{Analysis of Transcription Factors (TFs)}

TFs are key to plant growth, development, and the regulation of gene expression in response to abiotic and biotic stress. The top 20 TF families are shown at each stage in Fig. 6 and Table S7. Compared with GN, the TF families with the most DEGs in ProM were NAC, FAR1, and C2H2 (Fig. 6A). The top three TF families in the meiotic stage and the PostM stage were the same and included NAC, FAR1, and TARF (Fig. 6B and C). This was quite different from the previous three periods to anthesis, in which the TF families with the most DEGs were C2H2, FAR1, and bHLH (Fig. 6D). In addition, there was a significant difference in the number of DEGs corresponding to the upregulated and downregulated adjustments at each stage. There was little difference in the number of differentially expressed TF families in the four stages, ranging from 64 to 75 . The stage PostM had the highest numbers of DEGs, and the PreM stage had the lowest numbers of DEGs (Table S7).

\section{Analysis of gene expression patterns, DEG clustering, and functional enrichment}

According to the clustering heatmap analysis of the DEGs, the gene expression profiles underwent a series of changes between the different genotypes and/or at different developmental periods (Fig. 7A). According to the gene expression profile, the DEGs of two different genotypes at different developmental stages were clustered into 10 profiles (Fig. 7B and C). It was found that with time, the two genotypes exhibited differences in gene expression in response to spikelet growth and development. Profile $0,7,8$, and 9 were significantly overexpressed in LN ( $P<0.05$, Fig. 7B, Table S8). The difference was that Profile 0 , 8, and 9 were obviously overexpressed in $\mathrm{GN}(P<0.05)$, and Profile 7 was not overexpressed (Fig. 7C, Table S9). To determine the functional significance of the transcriptional changes of each genotype, GO and KEGG function enrichment analysis were performed on genes belonging to the overexpression profile (Fig. 7D-G). In LN, with the exception that response to hormone, gene silencing by RNA, and gene silencing were not enriched in Profile 0 , the remaining terms were all significantly enriched. There were significantly less terms enriched in Profile 7 and 9 than in Profile 0. Only protein binding in Profile 8 was significantly enriched (Fig. 7D). The KEGG enrichment results showed that RNA transport, RNA degradation, and phenylpropanoid biosynthesis were only significantly enriched in Profile 0. Zeatin synthesis, ribosome, and oxidative phosphorylation were only significantly enriched in Profile 9 . No pathway was enriched in Profile 0 and Profile 7 (Fig. 7E). In GN, RNA binding was significantly enriched in Profile 0 and Profile 8. Oxidoreductase activity, oxidation-reduction process, cell wall modification, and cell wall were significantly enriched in Profile 0 and Profile 9, and other terms were significantly enriched only in a single profile (Fig 7F). Pathway enrichment analysis showed that RNA transport was significantly enriched in Profile 0 and Profile 8. Phenylpropanoid biosynthesis and flavone and flavonol biosynthesis were significantly enriched in Profile 0. RNA degradation and DNA replication were significantly enriched in Profile 8. The remainder of the pathways were only significantly enriched in Profile 9 (Fig. 7G).

\section{Gene coexpression network analysis}

Weighted gene correlation network analysis (WGCNA) has a scale-free topological structure and is a systems biology method that is used to describe gene association patterns among different samples. It can be used to identify highly synergistic gene sets and candidate biomarker genes based on the interconnectivity of the gene sets and the association between the gene set and the phenotype. According to the pairwise correlation and gene expression trend of all samples, the unigene expression data from all 24 samples (two genotypes, three biological repeats, and four time points) were constructed into a coexpression network. The hierarchical clustering tree displays the coexpression module identified by WGCNA, and each leaf on the tree represents a gene. The main branch consisted of 18 modules (from a low serial number to a high serial number, M1 to M28), which are marked with different colors (Fig. 8A, Table S10). The 18 modules contained a total of 19981 unigenes, and the module with the least number of genes was midnightblue, which contained only 81 genes. The largest number of genes was in the turquoise module, which contained 4966 genes. The average number of genes per module was 1110 , and the genetic correlation between the modules was low (Fig. 8B, Table S10), which indicated that the analysis results of the module were reliable. In addition, to 
clarify the correlation between different modules, we also further conducted clustering and heatmap analysis. The two analyses exhibited the same result, both showing a high degree of correlation between the MEblue and MEred, MElightcyan and MEmidnightblue, MEgreen and MEyellow, MEcyan and MEgrey60, MEblack and MEpink, and MEgreenyellow and MEpurple modules (Fig. 8C, Table S11, S12). Finally, the correlation between the module and the sample was analyzed, and the result indicated that MEcyan and MEgrey60 had a high correlation with sample LA_2. In addition, MEsalmon, MEpink, and MEgreenyellow had a higher correlation with LPostM_1, GM_3, and GPostM_2, respectively (Fig. 8D, Table S13 and S14).

\section{qRT-PCR verification}

Sixteen genes possibly related to apomixis in Kentucky bluegrass were selected for qRT-PCR to verify the authenticity and reliability of the transcriptome results (Fig. 9, Table S15). The results indicated that the genes basically showed the same expression patterns and the same trend of change between the qRTPCR and RNA-seq results. However, the expression of CAT2 (catalase isozyme 2), which was downregulated at PostM in our results compared to GN, was not consistent with the RNA-seq results. Excluding the postmeiosis stage, the change trends in the premeiosis, meiosis, and anthesis stages were consistent with the RNA-seq results. The expression trend of the other 15 DEGs at four time points was the same as that of the RNA-seq results. Therefore, we conclude that the qRT-PCR results validated the transcriptional map data obtained from our RNA-seq analysis, confirming that the transcriptome sequencing results were accurate and reliable.

\section{Discussion}

\section{Embryological study on apomixis in Kentucky bluegrass}

Apomixis can fix any genotype across generations, and Kentucky bluegrass is a natural apomictic species closely related to major crops. The existing research on apomixis in Kentucky bluegrass has focused on various aspects, particularly cell embryology. In the late 1970s, Yong et al.[32] used the techniques of pistil cleaning and thick sectioning to observe the apomixis of Kentucky bluegrass. Subsequently, Marshall et al.[33] noted that the apomixis of Kentucky bluegrass was mainly aposporic. In addition, Kentucky bluegrass possesses a small number of parthenogenetic embryos, cocellular embryos, and antipodal embryos[11]. The occurrence of apospory in Kentucky bluegrass is caused by the expansion of one or more somatic cells in the ovules, which restores reproductive ability, and the unreduced embryo is then produced by several mitotic events[34,35]. The number of AICs is mostly one or two, though three are occasionally observed[36]. In this process, sexual embryos and apomictic embryos can coexist in the same ovule[37] and ultimately produce seeds with two or three embryos[11]. These previous reports support our research results, which demonstrated that during the embryonic development of Kentucky bluegrass, one or more nucellar cells located near the MMC under the nucellus epidermis became enlarged and exhibited an obvious nucleus, and their meristematic ability was restored. They then further developed to form AICs. The AICs developed into unreduced and unfertilized apomictic embryos, which could coexist with the sexual embryos.

\section{Transcriptome analysis of apomixis in Kentucky bluegrass}

Plant apomixis was first discovered in Alchornea ilicifolia in 1841[48]. Since this discovery, there has been much research on the classification, genetic evolution, embryonic development, morphogenesis, physiology, biochemistry, and breeding of apomixis[38, 39]. Researchers have long tried to collect natural apomixis resources and analyze their biological characteristics and genetic mechanisms, as well as introduce key genes into crops to make use of apomixis fixed heterosis in crops. However, due to the complexity of species, occurrence processes, and formation mechanisms, as well as the diversity in apomixis types, it is extremely difficult and time-consuming to mine and clone related genes using traditional breeding methods. Thus, a reverse genetic strategy based on transcriptomics, which is used to screen and identify candidate genes and then verify them by experimental mapping and functional analysis, has become the core of apomixis studies[23]. Furthermore, transcriptome methods are applicable to all types of plants and traits[40].

In this study, a reference transcriptome of wild germplasm materials of Kentucky bluegrass with significant differences in apomictic rates in Gansu, Chain, was constructed using RNA-seq, and the gene expression changes and differences between the two genotypes were studied. However, the ovary of Kentucky bluegrass is very small, and RNA is also very easily degraded, and thus the biological sample we used included the entire spikelet of Kentucky bluegrass, which is composed of 3-4 florets, including the glumes, lemma, palea, lodicules, pistil, and stamen. Therefore, our transcriptome originates from a variety of cell types, including somatic cells and male and female germ cells from the premeiosis to anthesis stages. Based on this, we believe that this database will be very helpful in identifying any transcripts expressed in the flowers of Kentucky bluegrass that can be identified at a detectable level. However, the spatial and temporal specificity of gene expression will need to be evaluated by other experiments such as qRT-PCR[23].

Regulation of TFs in the ovule development of apomixes: During plant growth and development, the expression of genes in cells is time-specific and spacespecific, which leads to differentiation and differences among different cells. One of the main reasons for this phenomenon is the regulation of TFs at the transcriptional level[41]. TFs are protein molecules that can specifically bind to cis-acting elements in the promoter region of eukaryotic genes to regulate the expression of target genes. They play an important role in plant growth and development, regulation, hormone signal transduction, and stress responses[42]. By comparing the transcripts of obligate apomixis and sexually reproductive Boechera plants, Shah et al.[43] showed that the dysregulation of TFs is one of the main components of apomixis-specific transcripts. Before this, scientists noted that no obvious developmental pathways or time changes could simply explain the shift from sexual reproduction to apomixis. The overexpression of TFs in apomixis-specific genes indicates a large-scale change in the regulation of apomixis ovules[44, 45]. Sharbel et al.[45] suggested that TFs are important motifs for the differential expression between sexual and apomictic ovules, and their mediation of the inhibition of gene expression is a characteristic of the development of sexual ovules to apomictic ovules. These studies suggest that TFs may regulate the transformation of plants from sexual reproduction to apomixis[46], and thus we further analyzed the differential expression of TFs in the two genotypes of Kentucky bluegrass. The results showed that a total of 84 TF families were differentially expressed in the two genotypes of Kentucky bluegrass, of which 52 were common across all four stages. 
Present studies on plant apomixis have reported that many TFs are involved in the regulation of apomixis. Kumar et al.[40] reported a transcriptome analysis of ovule development during nucellar embryogenesis in citrus (Citrus reticulata), and the results showed that MYB and WRKY were key TFs and were differentially expressed in single and polyembryonic ovules as well as between the pre-anthesis and post-anthesis stages of polyembryonic varieties. In addition, there are many reports that TFs such as B3[47], NAC and bZIP[43], MADS[45], OFP[46], TCP and Alfin-like[40] and bHLH, ARF and AUX/IAA[44] can regulate apomixis. These TFs have diverse functions and regulate plant responses to biotic and abiotic stresses in addition to many aspects of plant growth and development. Similarly, in our results, these TFs were differentially expressed in different genotypes and were time-specific, indicating that they may play an important role in regulating the apomixis development of Kentucky bluegrass. In addition, we also found that TFs such as FAR1, C2H2, TRAF, SWI/SNFBAF60b, and CPP were differentially expressed in different genotypes. They can regulate different genes and perform a variety of regulatory functions. For example, FAR1 can act as a Cdk inhibitor (CKI) to resist the transformation of G1/S, block cells in the G1 phase and then regulate the cell cycle[48]. It is speculated that they may be involved in the regulation of apomixis in Kentucky bluegrass. However, since our sampling included the whole spikelet, we suspect that many of the TFs we have screened may be involved in flower development, cell division, cell evolution, reproductive organ development, and so forth.

Disorder of meiosis leads to apomixes: The loss or failure of meiosis is one of the three basic events involved in apomixis[15]. Apomixis in Kentucky bluegrass is mainly aposporic, which is usually characterized by the failure of meiosis, followed by differentiation into one or more unreduced embryos from nucellus cells[49]. In Arabidopsis thaliana, apomixis also occurs through important meiotic genes, such as DYAD/SWITCH1 (SWI1), which controls meiotic chromosome tissue. Additionally, the MIME (mitosis instead of meiosis) of a triple mutant, which could be achieved in $A$. thaliana by combining different combinations of meiotic mutants spo11-1, osd1, and rec8, exhibits characteristics similar to diplospory[50,51]. Although the role of these genes or potential mutations in asexual reproduction in natural apomixis, such as in Boechera, has not been documented, a comparative MMC study between $A$. thaliana and Boechera indicated that meiotic genes in the MMC of Boechera such, as PARTING DANCERS and SPO11-2 (meiotic recombination protein), may be downregulated compared with those in the MMC of sexual A. thaliana[52]. Interestingly, Okada et al.[53] found that meiotic genes such as DMC1 (DNA meiotic recombinase 1), RAD50 (RAD50 double strand break repair protein), and other genes required for meiosis in A. thaliana, including SWITCH1/DYAD, MULTIPOLAR SPINDLE1, SPOROCYTELESS/ NOZZLE, REC8 (meiotic recombination protein) or similar transcripts, were not found in AIC, EAE, or SO cell types. It was previously reported that the meiosis control gene DMC1 was expressed in both sexual and apomixis MMCs in Hieracium spp., but could not be detected in Al cells by situ hybridization[54]. However, the results of this study indicated that these meiosis-related genes were differentially expressed between two genotypes, which may be due to the fact that our samples contained a variety of cellular tissues and originated from multiple time points. In addition, this discussion also implies the difference between diplospory and apospory. The former can be realized through loss of meiosis on the premise of avoiding fertilization, while the ability of nucellus cells to develop into mature embryo sacs through mitosis is more important for apospory.

Regulation of plant hormone signal transduction in apomixis development: Plant hormones directly or indirectly regulate the determination of cell fate, including embryogenesis and postembryonic development, and thus it is speculated that plant hormones may also affect the development of apomixis ovules[55]. In this study, many plant hormone-related genes were differentially expressed between the two genotypes, such as the auxin-related genes $S A U R$ (SAUR-like auxin-responsive protein family), $A R F$ (auxin response factor), IAA (auxin-responsive protein), $A U X 1$ (AUX transcriptional regulator family protein1), cytokinin synthesis-related genes IPT (tRNA dimethylallyltransferase 2), and CKX (cytokinin dehydrogenase 7), indicating that the expression of apospory in Kentucky bluegrass may be accompanied by a response to hormone stimulation. Giulio et al.[16] found that the DEGs between sexual reproduction and apomixis in Hypericum perforatum included genes involved in cytokinin biosynthesis, several auxin response factors, and SAUR-like proteins. After further analysis, it was noted that some genes related to hormone perception and dynamic balance, including cytokinin, auxin, and brassinol, were differentially expressed in the apomictic pistils. Schmidt et al.[52] reported that compared with mature gametophytes, the upregulation of cytokinin degradation genes was detected in apomictic genotypes, while the egg cell was marked by the activity of genes that lead to cytokinin modification. At the same time, it was found that the genes related to auxin signal transduction were enriched in AIC, but not expressed in sexual MMC[13]. We also determined the endogenous hormones of two genotypes and found that ZT and IAA may have significant effects on reproductive patterns. The change in the regularity of GA ${ }_{3}$ and $A B A$ was not obvious. We speculate that the differential expression of genes related to hormone signal transduction disrupts the balance of endogenous hormones in Kentucky bluegrass. The unbalanced hormone content affects plant morphogenesis, and so the two Kentucky bluegrass genotypes exhibit different reproductive patterns. In summary, our analysis of the results shows that the developmental pathway of apospory in Kentucky bluegrass is accompanied by the expression and regulation of multiple genes involved in hormone homeostasis.

Regulation of apomixis in Kentucky bluegrass by embryogenesis-related genes: The successful development of embryos is necessary in both sexual reproduction and apomixis. At present, it has been reported that SERK (somatic embryogenesis receptor-like kinase), LEC (laryngotracheo esophageal cleft), and WUS (WUSCHEL-related homeobox) are the main genes that regulate the development of apomixis. The expression of SERK is closely related to apomixis. Tucker et al.[56] studied the expression of AtSERK1 in sexual and apomictic Hieracium umbellatum, and the differential activity of SERK was also observed between sexual and asexual genotypes of Kentucky bluegrass[25]. In our study, the expression of SERK1 was upregulated in LN during the meiosis stage, while SERK2 was upregulated at the anthesis stage. WUS can promote the transformation from vegetative to embryogenic cells. In the absence of exogenous auxin, it binds with other genes that can induce embryo formation, such as $\angle E C 1, \angle E C 2$, and $S E R K 1$, to regulate the formation and development of embryos[57]. LEC regulates embryo formation by establishing an intercellular environment suitable for embryo development and is an important regulator for inducing embryo morphogenesis and controlling embryo development[58]. Compared with GN, we found that WUS1 and WDR23 (WD40 repeat-containing protein), as the homologous genes of $L E C 14 B$, were upregulated and time-specific. Comprehensive analysis showed that the upregulated expression of genes related to somatic and zygotic embryogenesis may regulate the occurrence of apomixis in Kentucky bluegrass.

Regulation of apomixis by the stress response pathway: One study showed that stress can induce the occurrence of somatic cell embryogenesis[59]. Many studies have observed a close correlation between stress-induced signal pathways and somatic embryogenesis induction[60-62]. One of the key steps in apospory is to confer nucellar cells embryogenesis ability. Our results showed that the stress response plays an important role in the development of apomixis 
in Kentucky bluegrass, and a large number of TFs related to stress responses, such as MYB, WRKY, and NAC, were differentially expressed between the two genotypes. In fact, many studies have found that genes involved in reproduction are associated with responses to stimulation and defense processes[63, 64]. Kumar et al.[40] found that many genes related to abiotic stress were significantly expressed in citrus polyembryos, including chalcone synthase, heat-shock factor protein, and chaperone protein CLPB1, indicating that the overexpression of stress-related signals was the main characteristic of the pre-anthesis ovules of citrus polyembryonic varieties. Shah et al.[43] also found that apomixis exhibited better tolerance to osmotic stress than sexual reproduction in vitro and was accompanied by the significant upregulation of a subset of NAC genes. These results indicate that stress response pathways are involved in early preparatory events before apomixis transition[23]. Previous studies hypothesized that the stress response pathway regulates apomixis due to prolonged UV exposure, which may be responsible for activating meiosis-specific proteins and initiating double-strand break formation and DNA repair, thus increasing the frequency of recombination[65,66].

Regulation of apomixis by epigenetic pathways and other factors: The genetics of apomixis is quite complex, often with large scale segregation aberrations[67]. Increasing studies have shown that mutations in the apparent pathway lead to phenotypes similar to apomixis phenotypes[68]. Studies on Paspalum notatum and Paspalumsimplex showed that the methylation level of genomic regions controlling apomixis was very high, and it was suggested that the relevant reproductive control factors were inactivated by methylation in Paspalum[69]. APOLLO (DNA cross-link repair 1B protein) is both an apomictic allele and a sexual allele[70], and so this gene has the potential to produce apomixis or sexual reproduction by regulation. In this study, $A P O L L O$ was upregulated compared with GN during the meiosis and postmeiosis stages, indicating that this gene regulates the development of apomixis in Kentucky bluegrass. In European rape (Brassica napus), the existence, deletion, and distribution of BnMET1a-like DNA methyltransferase genes are related to methylation, while methylation genes can transform pollen growth and development to embryogenesis, and the resulting embryo and zygotic embryo exhibit similar DNA methylation and MET1a-like expression patterns[71]. Our results also demonstrated that MET1 (methyltransferase 1) was differentially expressed between the two genotypes. Thus it can be seen that the regulation of epigenetics may be a key focus for the continued study of natural apomixis[68]. AGO9 can combine with different types of small RNAs to play a role in the regulation pf apomixis[72], and other genes involved in RNA-guided DNA methylation pathways also play important roles[73]. In parallel, according to a study that reported that small RNAs can promote DNA methylation in plants[74], including in maize, mutations in the effectors of the small RNA pathway have been shown to be important for the reproduction of events similar to diplospory[75]. In addition, some studies have shown that the mechanisms dependent on small RNAs play an important role in the cell specification within the ovule[76, 77]. Therefore, the regulatory mechanisms of epigenetics on apomixis in Kentucky bluegrass from a small RNA perspective should be explored in the future.

In addition, $S P L$ (squamosa-promoter binding protein-like protein)/NOZZLE, which is involved in the initiation of primary spore differentiation, also plays an important role in the regulation of apomixis[78, 79]. In this study, SPL 11 and $S P L 15$ were differentially expressed in the two genotypes, indicating that the expression of SPL/NOZZLE regulates the apomixis development of Kentucky bluegrass.

\section{Molecular regulation model of the apomixis mechanism of Kentucky bluegrass}

Based on the observation of the histological process of apomixis embryos and the study of gene regulation at the transcriptome level of wild germplasm materials of Kentucky bluegrass, we predicted the molecular regulatory model of apomixis embryogenesis in Kentucky bluegrass (Fig. 10). Compared with sexual reproduction, the apospory of Kentucky bluegrass avoids meiosis and double fertilization, which may be caused by temporal and spatial changes in related gene expression. Therefore, we speculate that the temporal and spatial changes in gene expression lead to the inhibition or imbalance of normal sexual reproduction in Kentucky bluegrass. As the plant needs to ensure the survival of offspring and produce seeds, it promotes the occurrence of apomixis. In essence, in facultative apomixis species such as Kentucky bluegrass, the occurrence of apomixis is mainly due to the disorder of the sexual reproductive pathway. The differential expression of stress response-related genes is extremely important for the acquisition of embryogenic cells and the change in hormone content. We speculate that the occurrence of events related to the stress response may be a preliminary preparation for apomixis in Kentucky bluegrass, which promotes the embryonic ability of the nucellus cells, causes a hormone imbalance, and leads to a disordered sexual reproductive pathway, which promotes the occurrence and development of apomixis. In summary, the regulatory mechanism of apomixis in Kentucky bluegrass is extremely complex, and its occurrence and development involve the coexpression of genes related to many aspects, such as the imbalance of meiosis, hormone signal transduction, embryonic development, stress response pathway, and epigenetic regulation pathway. In addition, we also found a number of new DEGs that have not been previously reported to be related to apomixis regulation, and so their apomixis regulatory mechanisms require further research.

\section{Conclusion}

The molecular basis of apomixis in Kentucky bluegrass was evaluated in detail by transcriptome analysis. In this study, the global gene expression of wild Kentucky bluegrass spikelets exhibiting significant differences in apomictic rates was compared, and many genes related to apomixis development were identified. The results showed that apomixis in Kentucky bluegrass was caused by changes in the expression of genes related to the sexual reproduction pathway, which led to disordered normal sexual reproduction, resulting in the production of offspring by apomixis. Through the functional enrichment analysis of DEGs, it was found that apomixis was regulated by TFs at the transcriptional level. In addition, it also involves the co-expression and co-regulation of genes related to meiosis, hormone signal transduction, embryonic development, the stress response pathway, and epigenetic pathway. Although this provides a basis for the molecular pathway of apomixis development, further studies on the genomic and functional characteristics are needed to elucidate the nature of its genetic determinants.

\section{Abbreviations}

LN: P. pratensis from Longnan; GN: P.pratensis form Gannan; RNA-seq: RNA sequencing; DEGs:Differentially expressed genes; KEGG: Kyoto Encyclopedia of Genes and Genomes; IAA: Indole-3-acetic acid; GA3: gibberellin; ABA: Abscisic acid; ZT: zeatin; NR: Non-Redundancy Protein; COG: Cluster of Orthologous 
Groups of proteins; GO: Gene Ontology; FPKM: The fragments per kb per million reads; FDR: False discovery rate; HPLC: High-performance liquid

chromatography; qRT-PCR: Quantitative Real-Time PCR

\section{Declarations}

Acknowledgements

We Thanks to Dr. Yi Shi for kindly providing the guidance of writing and Master Yan Liu for help of embryological analysis. We wish to thank all friends and colleagues who helped us in any way but are not included in the author list. We thank LetPub (www.letpub.com) for its linguistic assistance during the preparation of this manuscript.

Authors contributions

H.M. conceived the original research plans, supervised the experiments, provided funding supporting and agrees to serve as the author responsible for contact and ensures communication. J.Z. performed the experiments, analyzed the data and wrote the article.

Funding

This work was funded by the National Natural Science Foundation of China (NSFC) (project \# 31760699).

Availability of data and materials

Raw Illumina sequence data were deposited in the National Center for Biotechnology Information (NCBI) and be accessed in the sequence read archive (SRA) database (https://www.ncbi.nlm.nih.gov/sra) under accession numbers PRJNA680673. All data generated or analyzed during this study are included in this published article and the its supplementary materials files.

Ethics approval and consent to participate

Not applicable.

Consent for publication

Not applicable.

Competing interests

The authors declare that there are no conflict of interest.

\section{Author details}

Key Laboratory of Grassland Ecosystem of Ministry of Education, College of Grassland Science,

Gansu Agricultural University, Lanzhou 730070, China.

\section{References}

1. Hartley W: Studies on the origin, evolution and distribution of the Gramineae. IV. The genus Poa L. Australian Journal of Botany - AUST J BOT 1961, 9.

2. Soreng R: Chloroplast-DNA Phylogenetics and Biogeography in a Reticulating Group: Study in Poa (Poaceae). American Journal of Botany 1990, 77:13831400 .

3. Patterson J, Johnson P: Genome relationships in polyploid Poa pratensis and other Poa species inferred from phylogenetic analysis of nuclear and chloroplast DNA sequences. Genome / National Research Council Canada = Génome / Conseil national de recherches Canada 2005, 48:76-87.

4. McIntosh R: Breeding wheat for resistance to biotic stresses. Euphytica 1998, 100:19-34.

5. Mazzucato A, Barcaccia G, Pezzotti M, Falcinelli M: Biochemical and molecular markers for investigating the mode of reproduction in the facultative apomict Poa pratensis L. Sexual Plant Reproduction 1995, 8:133-138.

6. Nogler G: Gametophytic Apomixis. In.; 1984: 475-518.

7. Müntzing A: Apomictic and sexual seed formation in Poa. Hereditas 1933, 17(2):131-154.

8. Barcaccia G, Mazzucato A, Belardinelli A, Pezzotti M, Lucretti S, Falcinelli M: Inheritance of parental genomes in progenies of Poa pratensis L. from sexual and apomictic genotypes as assessed by RAPD markers and flow cytometry. Theoretical and Applied Genetics 1997, 95(4):516-524.

9. Wieners R, Fei S-Z, Johnson R: Characterization of a USDA Kentucky Bluegrass (Poa pratensis L.) Core Collection for Reproductive Mode and DNA Content by Flow Cytometry. Genetic Resources and Crop Evolution 2006, 53:1531-1541.

10. Matzk F, Prodanovic S, Bäumlein H, Schubert I: The Inheritance of apomixis in Poa pratensis confirms a five locus model with differences in gene expressivity and penetrance. The Plant cel/ 2005, 17(1):13-24.

11. Tian CC, Ma HL, Zhang YM: Embryo types and characteristics of apomixis in Poa pratensis L. Scientia Agricultura Sinica 2013, 46(13):2633-2642. 
12. Albertini E, Marconi G, Barcaccia G, Raggi L, Falcinelli M: Isolation of candidate genes for apomixis in Poa pratensis L. Plant molecular biology 2004, 56(6):879-894

13. Okada T, Hu Y, Tucker MR, Taylor JM, Johnson SD, Spriggs A, Tsuchiya T, Oelkers K, Rodrigues JC, Koltunow AM: Enlarging cells initiating apomixis in Hieracium praealtum transition to an embryo sac program prior to entering mitosis. Plant physiology 2013, 163(1):216-231

14. Albertini E, Barcaccia G, Porceddu A, Rosellini D, Falcinelli M: Genetic control of parthenogenesis in Poa pratensis L.: Results from a sexual $x$ apomictic cross. Proceedings of the XIX International grassland congress 2001:138-139.

15. Curtis M, Grossniklaus U: Molecular control of autonomous embryo and endosperm development. Sexual Plant Reproduction 2008, 21:79-88.

16. Galla G, Zenoni S, Avesani L, Altschmied L, Rizzo P, Sharbel TF, Barcaccia G: Pistil Transcriptome Analysis to Disclose Genes and Gene Products Related to Aposporous Apomixis in Hypericum perforatum L. Frontiers in plant science 2017, 8:79.

17. Sailer C, Schmid B, Grossniklaus U: Apomixis Allows the Transgenerational Fixation of Phenotypes in Hybrid Plants. Current Biology 2016, 26(3):331-337.

18. Francis G, Oliver J, Stevanato P, Sujatha M: Apomixis as a tool for development of high yielding clones and selections in Jatropha curcas L. Genetic Resources and Crop Evolution 2020, 67:727-743

19. Bicknell R, Koltunow A: Understanding Apomixis: Recent Advances and Remaining Conundrums. The Plant cel/ 2004,16 Suppl:S228-245.

20. Ozias-Akins P: Apomixis: Developmental characteristics and genetics. Critical Reviews in Plant Sciences 2006, 25(2):199-214.

21. Calzada JPV, Crane C, Stelly D: Botany - Apomixis: The asexual revolution. Science 1996, 274:1322-1323.

22. Mazzucato A, Falcinelli M, Veronesi F: Evolution and adaptedness in a facultatively apomictic grass, Poa pratensis L. Euphytica 1996, 92:13-19.

23. Ortiz J, Revale S, Siena L, Podio M, Delgado L, Stein J, Leblanc O, Pessino SJBg: A reference floral transcriptome of sexual and apomictic Paspalum notatum. 2017, 18(1):318.

24. Hecht V, Vielle-Calzada J, Hartog M, Schmidt E, Boutilier K, Grossniklaus U, de Vries SJPp: The Arabidopsis SOMATIC EMBRYOGENESIS RECEPTOR KINASE 1 gene is expressed in developing ovules and embryos and enhances embryogenic competence in culture. 2001, 127(3):803-816.

25. Albertini E, Marconi G, Reale L, Barcaccia G, Porceddu A, Ferranti F, Falcinelli MJPp: SERK and APOSTART. Candidate genes for apomixis in Poa pratensis. 2005, 138(4):2185-2199.

26. Lotan T, Ohto M, Yee KM, West MA, Lo R, Kwong RW, Yamagishi K, Fischer RL, Goldberg RB, Harada JJ: Arabidopsis LEAFY COTYLEDON1 is sufficient to induce embryo development in vegetative cells. Cell 1998, 93(7):1195-1205.

27. Zuo J, Niu QW, Frugis G, Chua NH: The WUSCHEL gene promotes vegetative-to-embryonic transition in Arabidopsis. The Plant journal : for cell and molecular biology 2002, 30(3):349-359.

28. Mercier R, Vezon D, Bullier E, Motamayor JC, Sellier A, Lefèvre F, Pelletier G, Horlow C: SWITCH1 (SWI1): A novel protein required for the establishment of sister chromatid cohesion and for bivalent formation at meiosis. Genes \& development 2001, 15:1859-1871.

29. Asker S, Jerling L: Apomixis in Plants. Boca Raton: CRC Press; 1992.

30. Barcaccia G, Albertini E: Apomixis in plant reproduction: a novel perspective on an old dilemma. Plant reproduction 2013, 26(3):159-179.

31. Huang DY: Gansu vegetation. Lanzhou, Gansu, GS; 1997.

32. Young BA, Sherwood R, Bashaw E: Cleared-pistil and thick-sectioning techniques for detecting aposporous apomixis in grasses. Canadian Journal of Botany-revue Canadienne De Botanique - CAN J BOT 1979, 57:1668-1672.

33. Marshall DR, Brown AHD: The evolution of apomixis. Heredity 1981, 47(1):1-15.

34. Holland J, Nyquist W, Cervantes-Martínez C: Plant Breeding Reviews. In., vol. 22; 2010: 9-112.

35. Miles J: Apomixis for Cultivar Development in Tropical Forage Grasses. Crop Science - CROP SCI 2007, 47.

36. Albertini E, Barcaccia G, Porceddu A, Sorbolini S, Falcinelli M: Mode of reproduction is detected by path1 and sex1 SCAR markers in a wide range of facultative apomictic Kentucky biuegrass varieties. Molecular Breeding 2001, 7:293-300.

37. Yahara K, Horie R, Kobayashi I, Sasaki A: Evolution of DNA double-strand break repair by gene conversion: coevolution between a phage and a restrictionmodification system. Genetics 2007, 176(1):513-526.

38. Czapik R, Jacobs SWL, Everett J: Apomixis in monocotyledons. Grasses: systematics and evolution. Collingwood : CSIRO Publishing 2000:316-321.

39. Ma SM, Wang YF, Ye XL, Zhao NX, Liang CY: Progress in study of apomixis in monocotyledonous plants. Chinese Bulletin of Botany 2002(5):530-537.

40. Kumar V, Malik S, Pal D, Srinivasan R, Bhat S: Comparative transcriptome analysis of ovules reveals stress related genes associated with nucellar polyembryony in citrus. Tree Genetics \& Genomes 2014, 10:449-464

41. Veenstra GJ, Wolffe AP: Gene-selective developmental roles of general transcription factors. Trends in biochemical sciences 2001, 26(11):665-671.

42. Tian C, Wan P, Sun S, Li J, Chen M: Genome-wide analysis of the GRAS gene family in rice and Arabidopsis. Plant molecular biology 2004, 54(4):519-532.

43. Shah J, Kirioukhova O, Pawar P, Tayyab M, Mateo J, Johnston A: Depletion of Key Meiotic Genes and Transcriptome-Wide Abiotic Stress Reprogramming Mark Early Preparatory Events Ahead of Apomeiotic Transition. Frontiers in plant science 2016, 7:1539.

44. Hofmann NR: Apomixis and Gene Expression in Boechera. The Plant cell 2010, 22:539.

45. Sharbel T, Voigt M, Corral J, Galla G, Kumlehn J, Klukas C, Schreiber F, Vogel H, Rotter BJTPc: Apomictic and sexual ovules of Boechera display heterochronic global gene expression pattems. 2010, 22(3):655-671

46. Tang Q, Zang G, Cheng C, Luan M, Dai Z, Xu Y, Yang Z, Zhao L, Su J: Diplosporous development in Boehmeria tricuspis: Insights from de novo transcriptome assembly and comprehensive expression profiling. Scientific Reports 2017, 7:46043. 
47. Galla G, Vogel H, Sharbel T, Barcaccia G: De novo sequencing of the Hypericum perforatum L. flower transcriptome to identify potential genes that are related to plant reproduction sensu lato. BMC Genomics 2015, 16:254.

48. Pope P, Bhaduri S, Pryciak PJCbC: Regulation of cyclin-substrate docking by a G1 arrest signaling pathway and the Cdk inhibitor Far1. 2014, 24(12):13901396.

49. Galla G, Barcaccia G, Schallau A, Puente Molins M, Bäumlein H, Sharbel TJSpr: The cytohistological basis of apospory in Hypericum perforatum L. 2011, 24(1):47-61.

50. Maruthachalam R, Marimuthu MPA, Siddiqi I: Gamete formation without meiosis in Arabidopsis. Nature 2008, 451:1121-1124.

51. d'Erfurth I, Jolivet S, Froger N, Catrice O, Novatchkova M, Mercier R: Turning Meiosis into Mitosis. PLoS biology 2009, 7:e1000124.

52. Schmidt A, Schmid M, Klostermeier U, Qi W, Guthörl D, Sailer C, Waller M, Rosenstiel P, Grossniklaus UJPg: Apomictic and sexual germline development differ with respect to cell cycle, transcriptional, hormonal and epigenetic regulation. 2014, 10(7):e1004476.

53. Okada T, Catanach A, Johnson S, Bicknell R, Koltunow A: An Hieracium mutant, loss of apomeiosis 1 (loa1) is defective in the initiation of apomixis. Sexual Plant Reproduction 2007, 20:199-211

54. Couteau F, Belzile F, Horlow C, Grandjean O, Vezon D, Doutriaux MJTPc: Random chromosome segregation without meiotic arrest in both male and female meiocytes of a dmc1 mutant of Arabidopsis. 1999, 11(9):1623-1634.

55. Tucker M, Okada T, Johnson S, Takaiwa F, Koltunow AJJoeb: Sporophytic ovule tissues modulate the initiation and progression of apomixis in Hieracium 2012, 63(8):3229-3241.

56. Tucker M, Araujo A, Paech N, Hecht V, Schmidt E, Rossell J, De Vries S, Koltunow AJTPc: Sexual and apomictic reproduction in Hieracium subgenus pilosella are closely interrelated developmental pathways. 2003, 15(7):1524-1537.

57. Braybrook S, Stone S, Park S, Bui A, Le B, Fischer R, Goldberg R, Harada JJPotNAoSotUSoA: Genes directly regulated by LEAFY COTYLEDON2 provide insight into the control of embryo maturation and somatic embryogenesis. 2006, 103(9):3468-3473.

58. Kwong R, Bui A, Lee H, Kwong L, Fischer R, Goldberg R, Harada JJTPc: LEAFY COTYLEDON1-LIKE defines a class of regulators essential for embryo development. 2003, 15(1):5-18.

59. Bräuning S, Catanach A, Lord J, Bicknell R, Macknight RJBpb: Comparative transcriptome analysis of the wild-type model apomict Hieracium praealtum and its loss of parthenogenesis (lop) mutant. 2018, 18(1):206.

60. Jin F, hu L, Yuan D, Xu J, Gao W, He L, Yang X, Zhang X: Comparative transcriptome analysis between somatic embryos (SEs) and zygotic embryos in cotton: Evidence for stress response functions in SE development. Plant biotechnology journal 2014, 12:161-173.

61. Salvo S, Hirsch C, Buell C, Kaeppler S, Kaeppler HJPo: Whole transcriptome profiling of maize during early somatic embryogenesis reveals altered expression of stress factors and embryogenesis-related genes. 2014, 9(10):e111407.

62. Wickramasuriya A, Dunwell JJBg: Global scale transcriptome analysis of Arabidopsis embryogenesis in vitro. 2015, 16:301.

63. Zhou L, Dresselhaus T: Friend or foe: Signaling mechanisms during double fertilization in flowering seed plants. In.; 2018.

64. Wyder S, Rivera A, Valdés A, Cañal M, Gagliardini V, Fernández H, Grossniklaus UJPp, PPB b: Differential gene expression profiling of one- and twodimensional apogamous gametophytes of the fern Dryopteris affinis ssp. affinis. 2020, 148:302-311.

65. Hörandl E, Hadacek F: The oxidative damage initiation hypothesis for meiosis. Plant reproduction 2013, 26:351-367.

66. Klatt S, Hadacek F, Hodač L, Brinkmann G, Eilerts M, Hojsgaard D, Hörandl EJFips: Photoperiod Extension Enhances Sexual Megaspore Formation and Triggers Metabolic Reprogramming in Facultative Apomictic Ranunculus auricomus. 2016, 7:278.

67. Abdi S, Prasad S, Dwivedi A, Bhat V: Harnessing Apomixis for Heterosis Breeding in Crop Improvement. 2016, 11:79-99.

68. Hand M, Koltunow A: The Genetic Control of Apomixis: Asexual Seed Formation. Genetics 2014, 197:441-450.

69. Podio M, Cáceres M, Samoluk S, Seijo J, Pessino S, Ortiz J, Pupilli FJJoeb: A methylation status analysis of the apomixis-specific region in Paspalum spp. suggests an epigenetic control of parthenogenesis. 2014, 65(22):6411-6424.

70. Corral J, Vogel H, Aliyu O, Hensel G, Thiel T, Kumlehn J, Sharbel T: A Conserved Apomixis-Specific Polymorphism Is Correlated with Exclusive Exonuclease Expression in Premeiotic Ovules of Apomictic Boechera Species. Plant physiology 2013, 163:1660-1672.

71. Solís M, Rodríguez-Serrano M, Meijón M, Cañal M, Cifuentes A, Risueño M, Testillano PJJoeb: DNA methylation dynamics and MET1a-like gene expression changes during stress-induced pollen reprogramming to embryogenesis. 2012, 63(18):6431-6444.

72. Meister G: Meister, G. Argonaute proteins: functional insights and emerging roles. Nature reviews Genetics 2013, 14:447-459.

73. Hernández-Lagana E, Rodriguez-Leal D, Lúa J, Vielle-Calzada J-P: A Multigenic Network of ARGONAUTE4 Clade Members Controls Early Megaspore Formation in Arabidopsis. Genetics 2016, 204:1045-1056.

74. Bao N, Lye K, Barton MJDc: MicroRNA binding sites in Arabidopsis class III HD-ZIP mRNAs are required for methylation of the template chromosome. 2004, 7(5):653-662.

75. Singh M, Goel S, Meeley R, Dantec C, Parrinello H, Michaud C, Leblanc O, Grimanelli DJTPc: Production of viable gametes without meiosis in maize deficient for an ARGONAUTE protein. 2011, 23(2):443-458.

76. Martinez G, Köhler CJCoipb: Role of small RNAs in epigenetic reprogramming during plant sexual reproduction. 2017, 36:22-28.

77. Gehring MJTNp: Epigenetic dynamics during flowering plant reproduction: evidence for reprogramming? 2019, 224(1):91-96.

78. Yang W, Ye D, Xu J, Sundaresan VJG, development: The SPOROCYTELESS gene of Arabidopsis is required for initiation of sporogenesis and encodes a novel nuclear protein. 1999, 13(16):2108-2117

Page $11 / 24$ 
79. Balasubramanian S, Schneitz KJD: NOZZLE regulates proximal-distal pattern formation, cell proliferation and early sporogenesis during ovule development in Arabidopsis thaliana. 2000, 127(19):4227-4238.

80. Liu Y, Zhang JQ, Niu KJ, Dong WK, Ma HL, Li YZ: Identification of apomictic characteristics of wild Kentucky bluegrass germplasm Resources in Gansu. Grassland and Turf 2020, 40(3):84-89.

81. Grabherr MG, Haas BJ, Yassour M, Levin JZ, Thompson DA, Amit I, Adiconis X, Fan L, Raychowdhury R, Zeng Q et al: Full-length transcriptome assembly from RNA-Seq data without a reference genome. Nature biotechnology 2011, 29(7):644-652.

82. Fu L, Niu B, Zhu ZW, Wu ST, Li WZ: CD-HIT: accelerated for clustering the next generation sequencing data. BIOINFORMATICS 2012, 28(23):3150-3152. 83. Li B, Dewey CN: RSEM: accurate transcript quantification from RNA-Seq data with or without a reference genome. BMC bioinformatics $2011,12: 323$.

84. Anders S, Huber W: Differential expression analysis for sequence count data. Genome biology 2010, 11(10):R106.

85. Benjamini Y, Hochberg Y: Controlling The False Discovery Rate - A Practical And Powerful Approach To Multiple Testing. Journal of the Royal Statal Society Series B: Methodological 1995, 57:289-300.

86. Young MD, Wakefield MJ, Smyth GK, Oshlack A: Gene ontology analysis for RNA-seq: accounting for selection bias. Genome biology 2010,11 (2):R14.

87. Kanehisa M, Araki M, Goto S, Hattori M, Hirakawa M, Itoh M, Katayama T, Kawashima S, Okuda S, Tokimatsu T et al: KEGG for linking genomes to life and the environment. Nucleic acids research 2008, 36(Database issue):D480-484.

88. Livak K, Schmittgen T: Analysis of Relative Gene Expression Data using Real-Time Quantitative PCR. Methods (San Diego, Calif) 2002, 25:402-408.

\section{Tables}

Table 1 RNA-seq data quality summary

\begin{tabular}{|llllllll|}
\hline Sample & Raw Reads & Clean Reads & Clean Bases & Error(\%) & Q20(\%) & Q30(\%) & GC Content(\%) \\
\hline LPreM & 23595847.33 & 22667952 & $6.80 G$ & 0.023 & 98.13 & 94.48 & 53.30 \\
\hline LM & 23046213.33 & 21703867 & $6.51 G$ & 0.02 & 98.12 & 94.46 & 52.17 \\
\hline LPostM & 22725789 & 21366863.67 & $6.41 G$ & 0.02 & 98.26 & 94.76 & 52.28 \\
\hline LA & 22815332.67 & 21630936.33 & $6.49 G$ & 0.02 & 98.32 & 94.89 & 54.06 \\
\hline GPreM & 22034463 & 20955782 & $6.29 G$ & 0.02 & 98.32 & 94.88 & 53.42 \\
\hline GM & 22695871.33 & 21666821.33 & $6.50 G$ & 0.02 & 98.31 & 94.86 & 53.52 \\
\hline GPostM & 22588325 & 21336127.67 & $6.40 G$ & 0.02 & 98.24 & 94.71 & 53.41 \\
\hline GA & 22232579.33 & 20733056.33 & $6.22 G$ & 0.02 & 98.17 & 94.54 & 53.75 \\
\hline
\end{tabular}

Table 2 Number of DEGs exhibiting upregulated or downregulated expression at four stages in LN and GN

\begin{tabular}{|c|c|c|c|c|c|c|c|c|c|c|c|c|}
\hline & $\begin{array}{l}\text { LAvs. } \\
\text { LPostM }\end{array}$ & LAvs.LM & $\begin{array}{l}\text { LAvs. } \\
\text { LPreM }\end{array}$ & $\begin{array}{l}\text { LPostM } \\
\text { vs.LM }\end{array}$ & $\begin{array}{l}\text { LPostM } \\
\text { vs.LPreM }\end{array}$ & $\begin{array}{l}\text { LMvs. } \\
\text { LPreM }\end{array}$ & $\begin{array}{l}\text { GAvs. } \\
\text { GPostM }\end{array}$ & GAvs.GM & $\begin{array}{l}\text { GAvs. } \\
\text { GPreM }\end{array}$ & $\begin{array}{l}\text { GPostM } \\
\text { vs.GM }\end{array}$ & $\begin{array}{l}\text { GPostM } \\
\text { vs.GPreM }\end{array}$ & $\begin{array}{l}\text { GMvs. } \\
\text { GPreM }\end{array}$ \\
\hline Downregulated & 11194 & 15046 & 19091 & 147 & 7675 & 7758 & 11469 & 19208 & 26373 & 2861 & 26470 & 8296 \\
\hline Upregulated & 13521 & 11802 & 11849 & 176 & 2037 & 10193 & 10660 & 19619 & 17896 & 5925 & 15794 & 18512 \\
\hline Total & 24715 & 26848 & 30940 & 323 & 9712 & 17951 & 22129 & 38827 & 44269 & 8786 & 42264 & 26808 \\
\hline
\end{tabular}

Table 3 Analysis of the KEGG enrichment of the DEGs between LN and GN. 


\begin{tabular}{|c|c|c|c|c|c|c|c|c|}
\hline \multirow[b]{2}{*}{ pathway } & \multicolumn{2}{|c|}{ LPreM vs. GPreM } & \multicolumn{2}{|c|}{ LPostM vs. GPostM } & \multicolumn{2}{|c|}{ LM vs. GM } & \multicolumn{2}{|c|}{ LM vs. GM } \\
\hline & $\begin{array}{l}\text { Gene } \\
\text { number }\end{array}$ & P-Value & $\begin{array}{l}\text { Gene } \\
\text { number }\end{array}$ & P-Value & $\begin{array}{l}\text { Gene } \\
\text { number }\end{array}$ & P-Value & $\begin{array}{l}\text { Gene } \\
\text { number }\end{array}$ & P-Value \\
\hline DNA replication & 61 & 0.001565 & 60 & 0.990152 & 77 & 0.575397 & 52 & 0.112859 \\
\hline Phenylpropanoid biosynthesis & 115 & 0.007247 & 203 & 0.121084 & 147 & 0.986556 & 67 & 0.999233 \\
\hline Homologous recombination & 52 & 0.067711 & 56 & 0.999108 & 63 & 0.978747 & 44 & 0.566804 \\
\hline Oxidative phosphorylation & 71 & 0.202885 & 148 & 0.105614 & 126 & 0.526184 & 68 & 0.572862 \\
\hline RNA degradation & 77 & 0.404024 & 143 & 0.799797 & 146 & 0.572418 & 74 & 0.788342 \\
\hline Linoleic acid metabolism & 12 & 0.532578 & 23 & 0.64896 & 26 & 0.365923 & 8 & 0.938033 \\
\hline Betalain biosynthesis & 1 & 0.69271 & 6 & 0.065747 & 4 & 0.247367 & 1 & 0.721767 \\
\hline Peroxisome & 46 & 0.829438 & 99 & 0.820379 & 109 & 0.368462 & 69 & 0.086475 \\
\hline Flavone and flavonol biosynthesis & 1 & 0.915212 & 13 & 0.009109 & 7 & 0.275795 & 4 & 0.317912 \\
\hline mRNA surveillance pathway & 62 & 0.923683 & 132 & 0.948748 & 139 & 0.747065 & 63 & 0.97737 \\
\hline RNA polymerase & 16 & 0.927529 & 28 & 0.996929 & 34 & 0.943184 & 24 & 0.563707 \\
\hline Cell cycle - Caulobacter & 6 & 0.93406 & 21 & 0.543922 & 18 & 0.714516 & 8 & 0.855941 \\
\hline RNA transport & 87 & 0.960775 & 170 & 0.998862 & 228 & 0.115334 & 95 & 0.964279 \\
\hline Basal transcription factors & 18 & 0.972548 & 42 & 0.972845 & 34 & 0.997506 & 24 & 0.870803 \\
\hline Plant hormone signal transduction & 73 & 0.995713 & 224 & 0.104245 & 189 & 0.681502 & 83 & 0.991174 \\
\hline Zeatin biosynthesis & 1 & 0.999943 & 23 & 0.390375 & 16 & 0.841514 & 8 & 0.855941 \\
\hline$A B C$ transporters & 13 & 0.985709 & 41 & 0.795381 & 42 & 0.674365 & 19 & 0.8837 \\
\hline $\begin{array}{l}\text { Amino sugar and nucleotide sugar } \\
\text { metabolism }\end{array}$ & 71 & 0.425583 & 191 & 0.000298 & 128 & 0.776029 & 60 & 0.962007 \\
\hline Ribosome & 245 & $7.55 \mathrm{E}-06$ & 190 & 1 & 198 & 1 & 244 & 0.001101 \\
\hline
\end{tabular}

Table 4 Meteorological information and apomictic rates of Kentucky bluegrass

\begin{tabular}{|lllclcc|}
\hline $\begin{array}{l}\text { Germplasm } \\
\text { materials }\end{array}$ & Collection site & Habitat & Altitude/m & $\begin{array}{l}\text { Average annual } \\
\text { rainfall/mm }\end{array}$ & $\begin{array}{c}\text { Average annual temperature/ } \\
{ }^{\circ} \mathrm{C}\end{array}$ & $\begin{array}{l}\text { Apomictic } \\
\text { rate/\% }\end{array}$ \\
\hline LN & $\begin{array}{l}\text { Longnan, } \\
\text { Gansu }\end{array}$ & Hillside & 1578.0 & 536.0 & 8.4 \\
\hline GN & Gannan, Gansu & $\begin{array}{l}\text { Alpine } \\
\text { meadow }\end{array}$ & 3143.0 & 514.0 & 2.6 \\
\hline
\end{tabular}

Table 5 Information of planting sites of wild germplasm materials of Kentucky bluegrass

\begin{tabular}{|llllllllrr}
\hline Altitude/m & $\begin{array}{l}\text { Annual } \\
\text { precipitation/mm }\end{array}$ & $\begin{array}{l}\text { Annual } \\
\text { evaporation/mm }\end{array}$ & $\begin{array}{l}\text { Annual } \\
\text { sunshine } \\
\text { hours/h }\end{array}$ & $\begin{array}{l}\text { Annual } \\
\text { mean } \\
\text { temperature/ } \\
{ }^{\circ} \mathrm{C}\end{array}$ & $\begin{array}{l}\text { Frost- } \\
\text { free } \\
\text { period/d }\end{array}$ & $\begin{array}{l}\text { Organic } \\
\text { matter/\% }\end{array}$ & $\begin{array}{l}\text { Total } \\
\text { nitrogen/\% }\end{array}$ & $\begin{array}{l}\text { Available } \\
\text { nitrogen/\% }\end{array}$ & $\begin{array}{l}\text { Available } \\
\text { potassium/\% }\end{array}$ \\
\hline 1517.30 & 350.00 & 1664.00 & 2474.40 & 10.30 & 171.00 & 2.83 & 0.09 & 49.88 & 173.19 \\
\hline
\end{tabular}

\section{Figures}




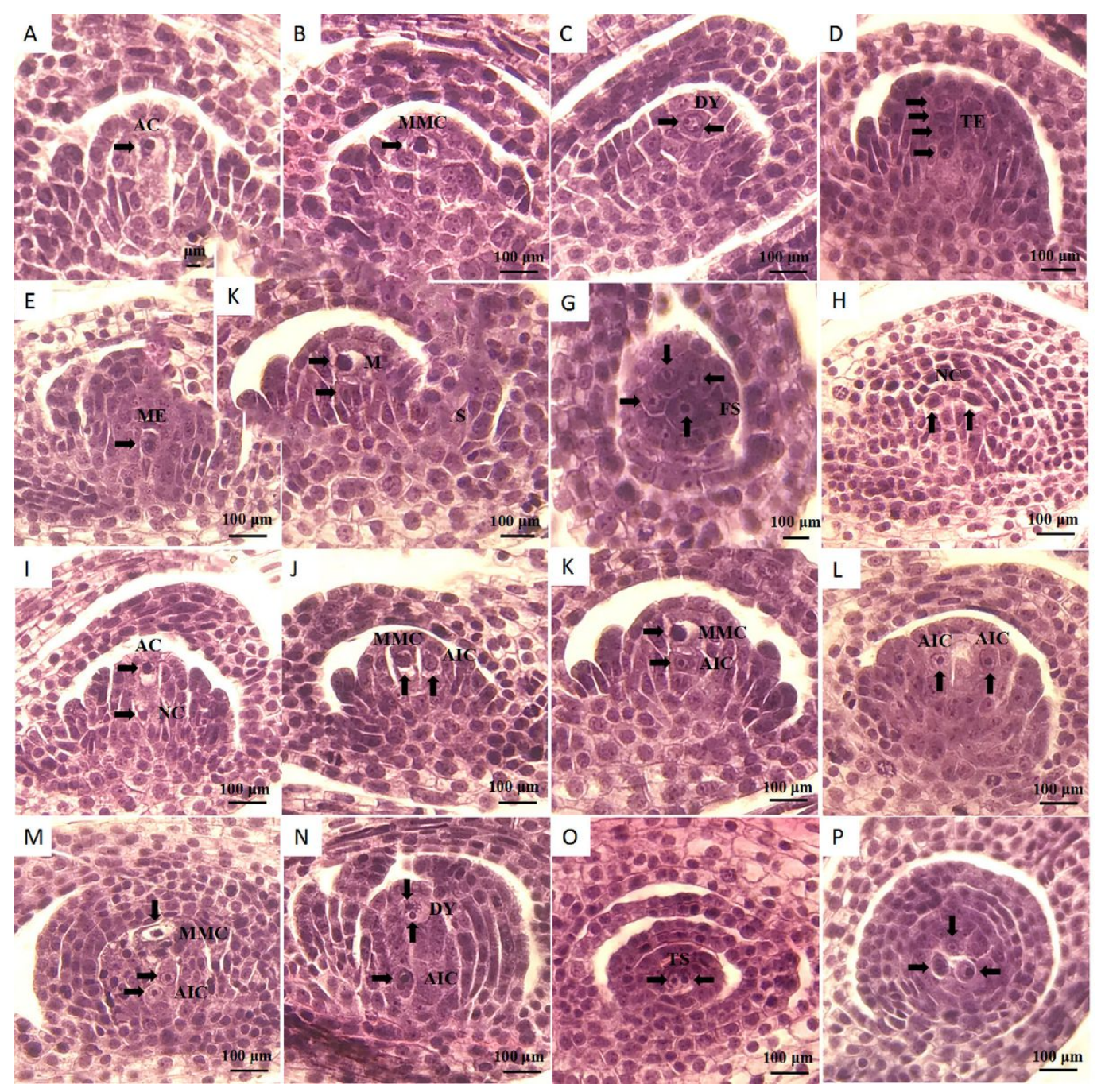

Figure 1

Cytoembryological observation of the sexual ovules and apomixis ovules of Kentucky bluegrass A- $G$ indicate the development of the sexual reproductive embryo sac. A: the stage of the AC, B: the stage of the MMC, C: the stage of the DY, D: the stage of the TE, E: the stage of the ME, F: the stage of the TS, G: the stage of the FS. H-P represent the observation of AICs. H: the period of specialized NC, I: the coexistence period of AC and specialized NC, J and K: the period of coexistence of MMC and AIC, L: the coexistence period of two AICs, M: the coexistence period of two AICs and MMC, N: the period of coexistence of AIC and DY; O: the stage of the TS of AIC; P: the period of the three-nucleus embryo sac. AC: archesporial cell; MMC: megaspore mother cell; DY: dyad; TE: tetrad; ME: megasporangium; TS: two-nucleus embryo sac; FS: four-nucleus embryo sac; NC: nucellar cell; AIC: aposporous initiating cell. 

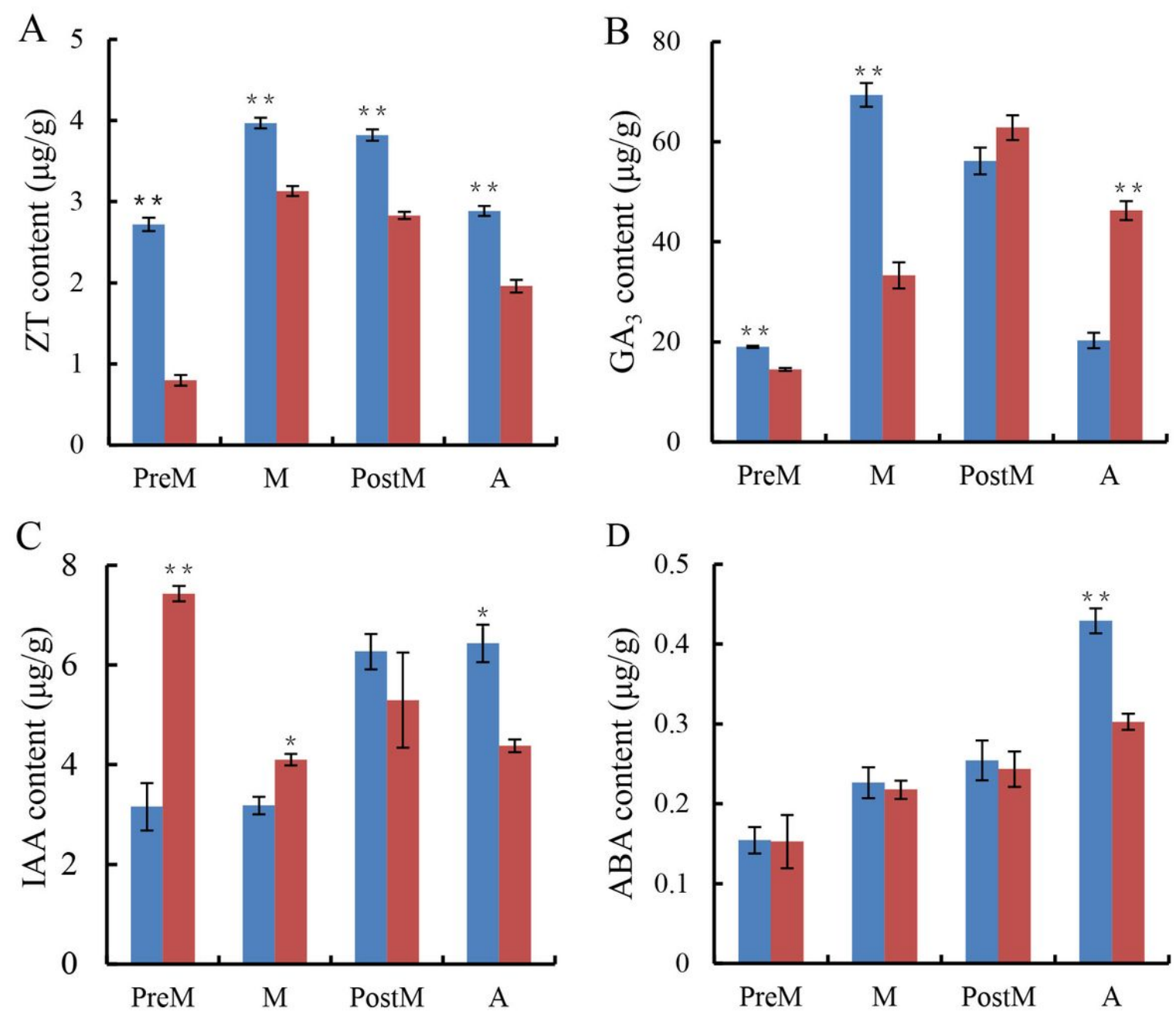

\section{Figure 2}

Changes in endogenous hormone content in the spikelets of Kentucky bluegrass Spikelet endogenous hormone content in LN (blue column) and GN (red

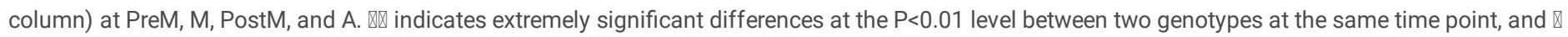
indicates significant differences at the $\mathrm{P}<0.05$ level. 
A

\begin{tabular}{ccc}
\hline & Transcripts & Unigenes \\
\hline min length (nt) & 301 & 301 \\
mean length (nt) & 830 & 801 \\
median length (nt) & 640 & 585 \\
max length (nt) & 13045 & 13045 \\
N50 & 987 & 969 \\
N90 & 428 & 400 \\
total nucleotides & 741017724 & 231171117 \\
total numbers & 892774 & 288678 \\
\hline
\end{tabular}

C

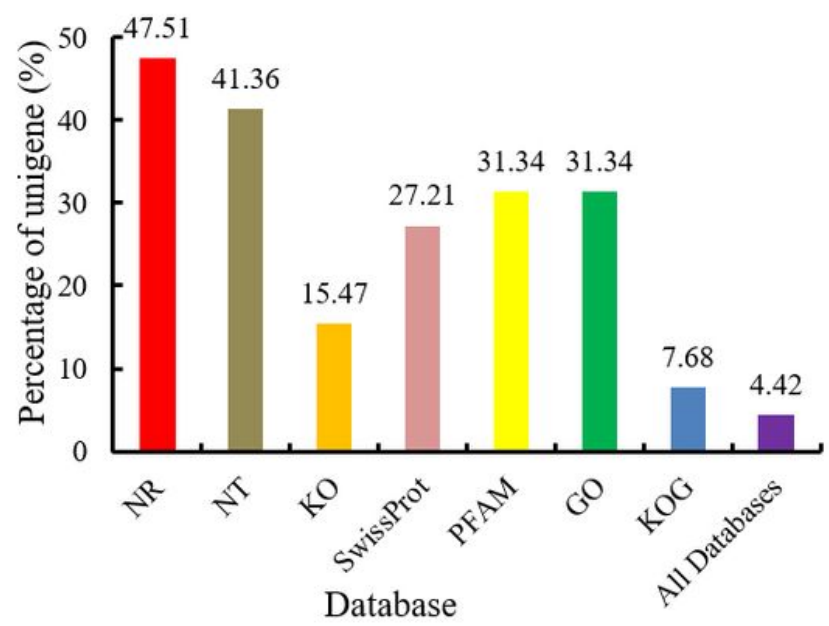

B

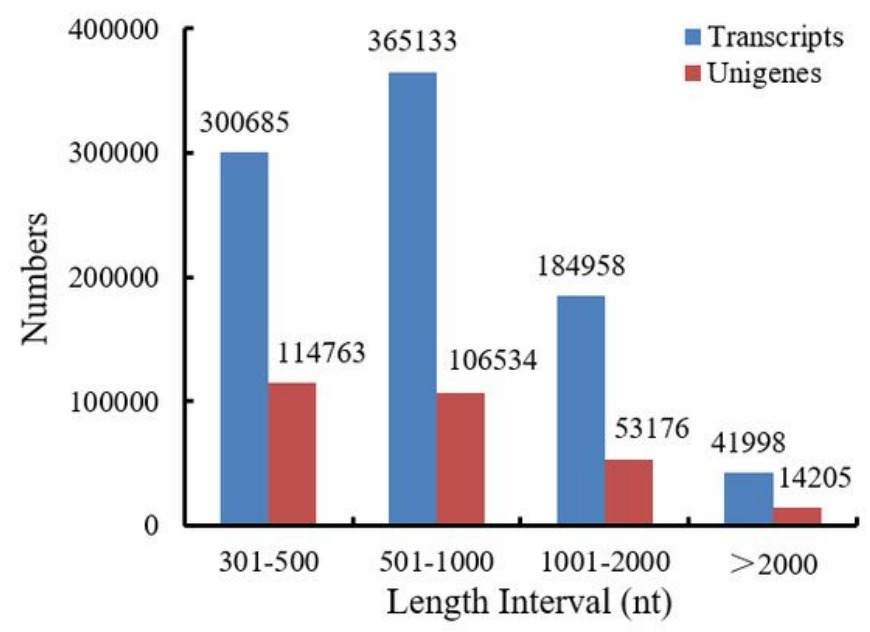

D

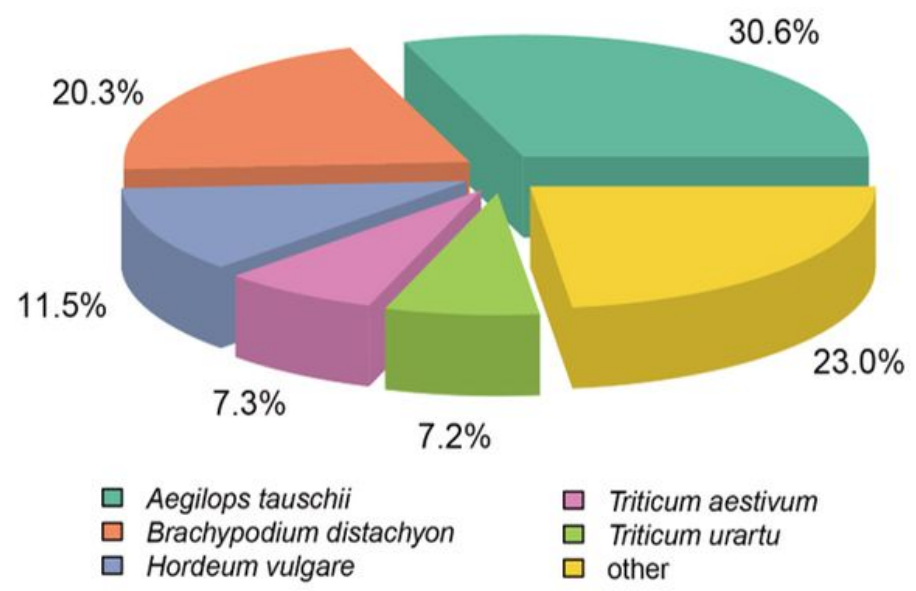

\section{Figure 3}

Assembly data and functional notes of the transcriptome. A: Length and quantity statistics of the transcripts and unigenes. B: Length distribution of transcripts and unigenes. C: Annotation results for different database functions. D: Homologous plant species in the $\mathrm{Nr}$ database. 

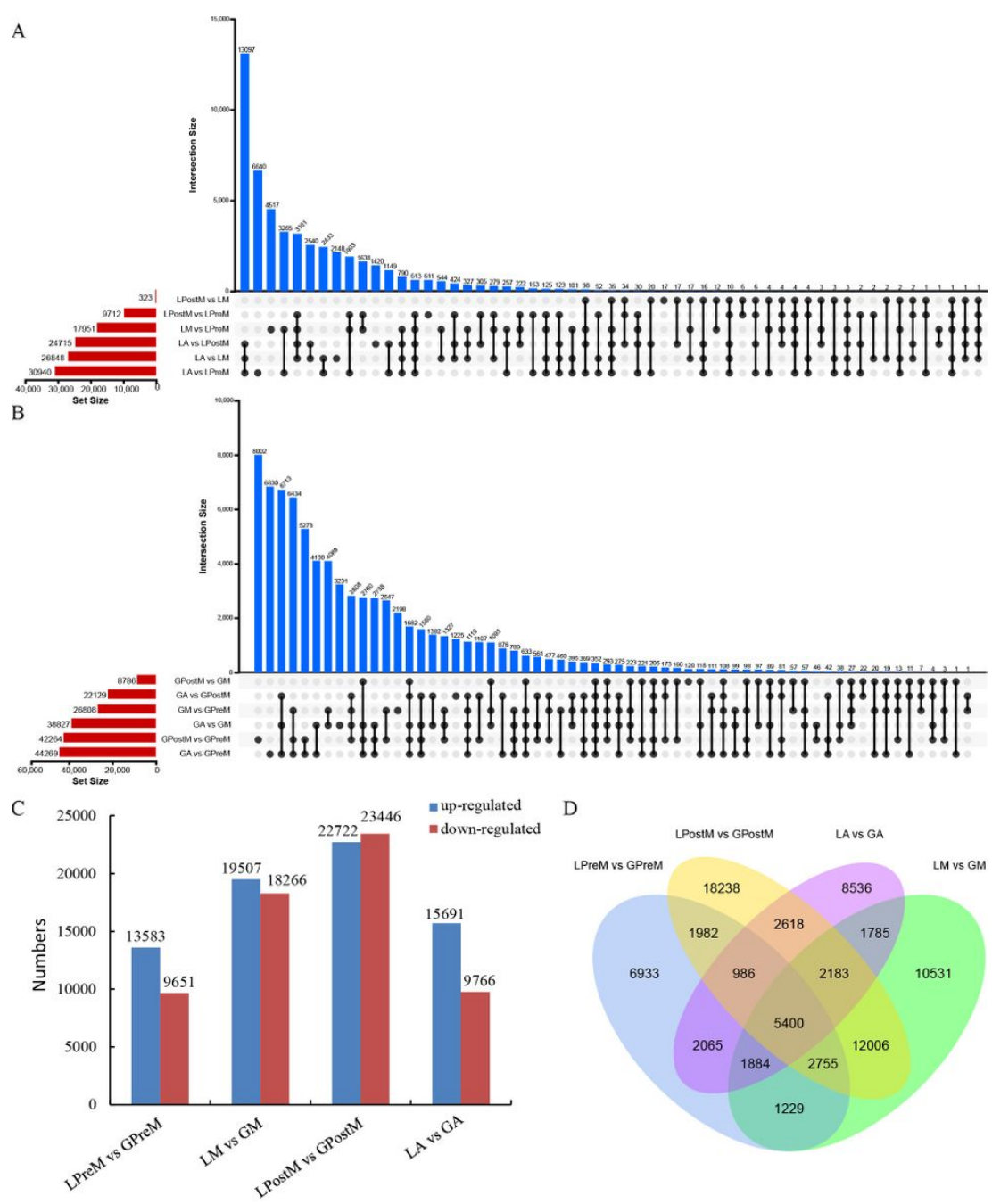

\section{Figure 4}

DEGs identified at four stages in LN and GN. A: DEG identification and quantitative statistics in LN. B: DEG identification and quantitative statistics in LN. C: Number of DEGs exhibiting upregulated or downregulated expression at four stages in LN vs. GN. D: Venn diagrams of all genes between LN and GN. 


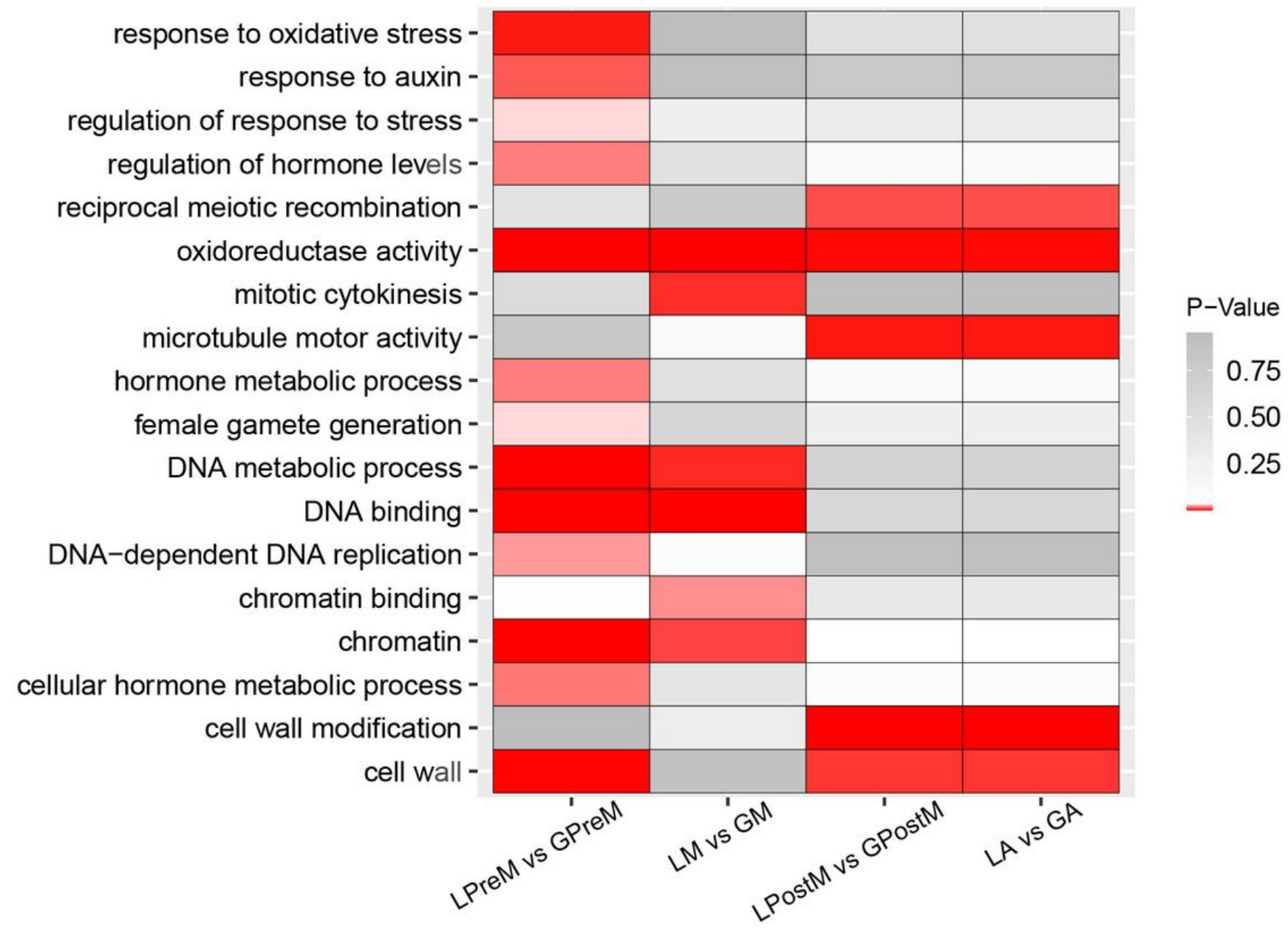

Figure 5

Analysis of the GO enrichment of the DEGs between LN and GN. 
A

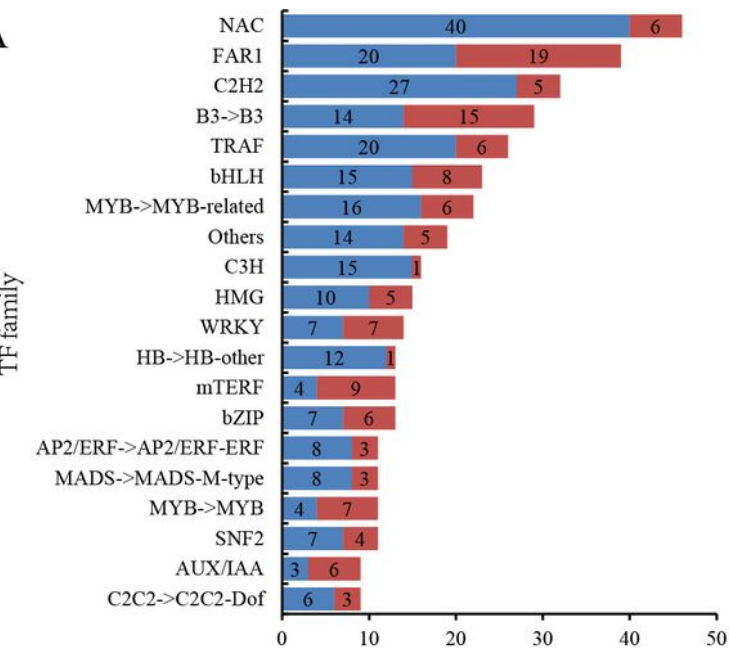

C

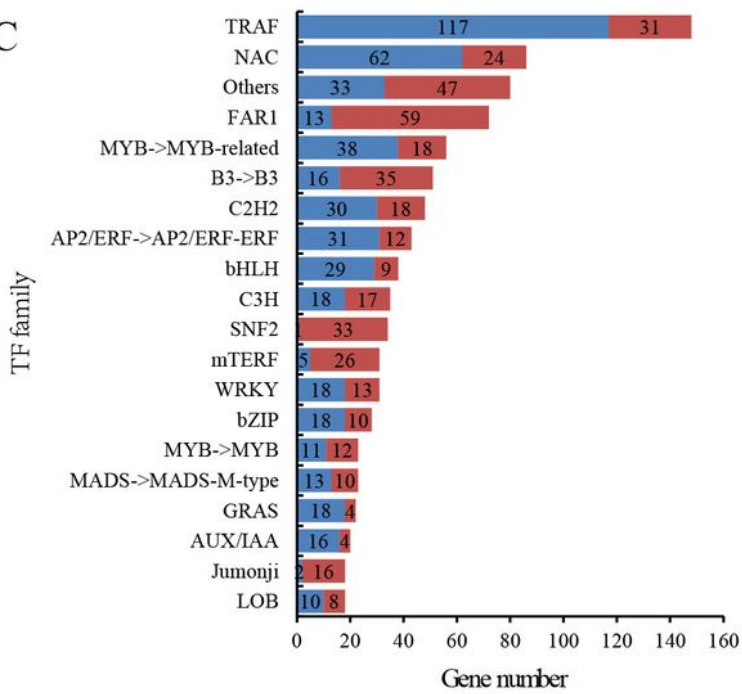

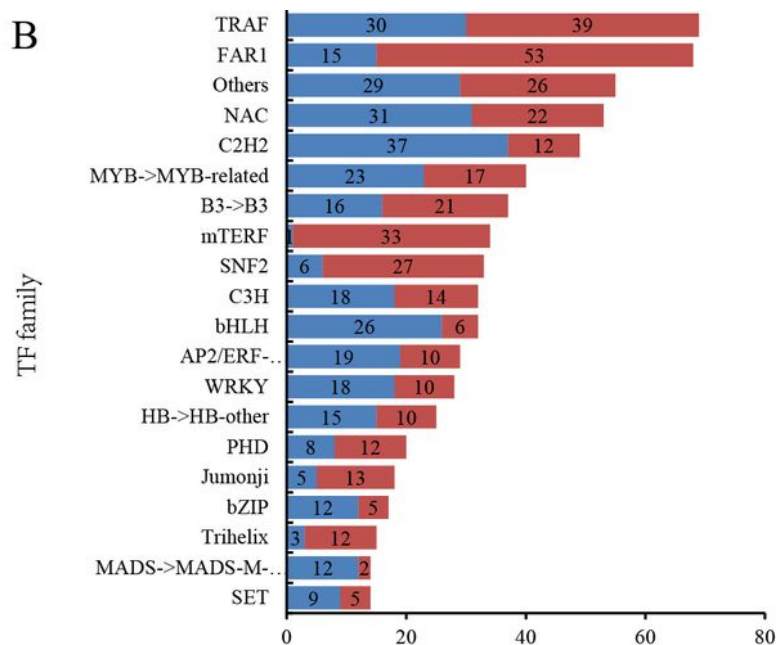

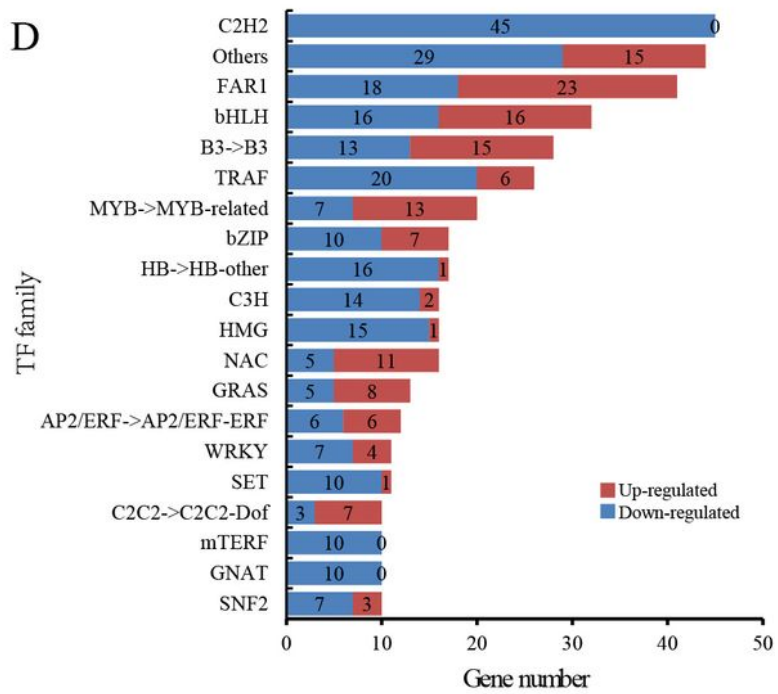

Figure 6

Analysis of TFs differentially expressed between LN and GN. A: LPreM vs. GPreM. B: LM vs. GM. C: LPostM vs. GPostM. D: LA vs. GA. 


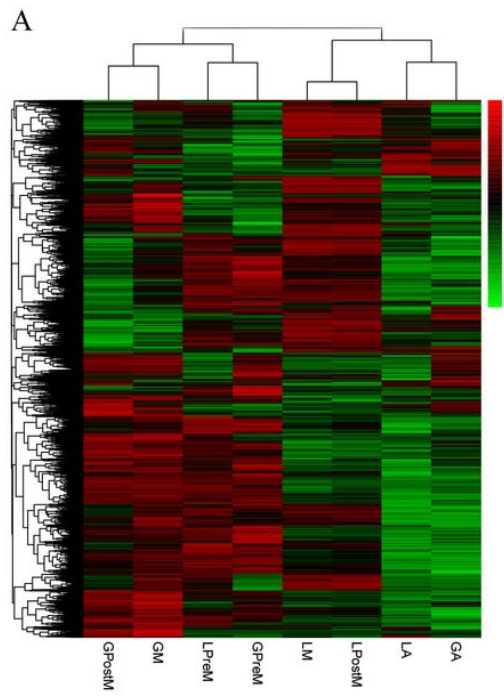

B
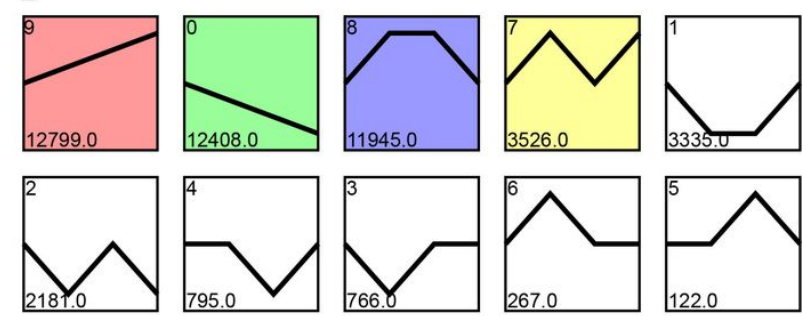

C
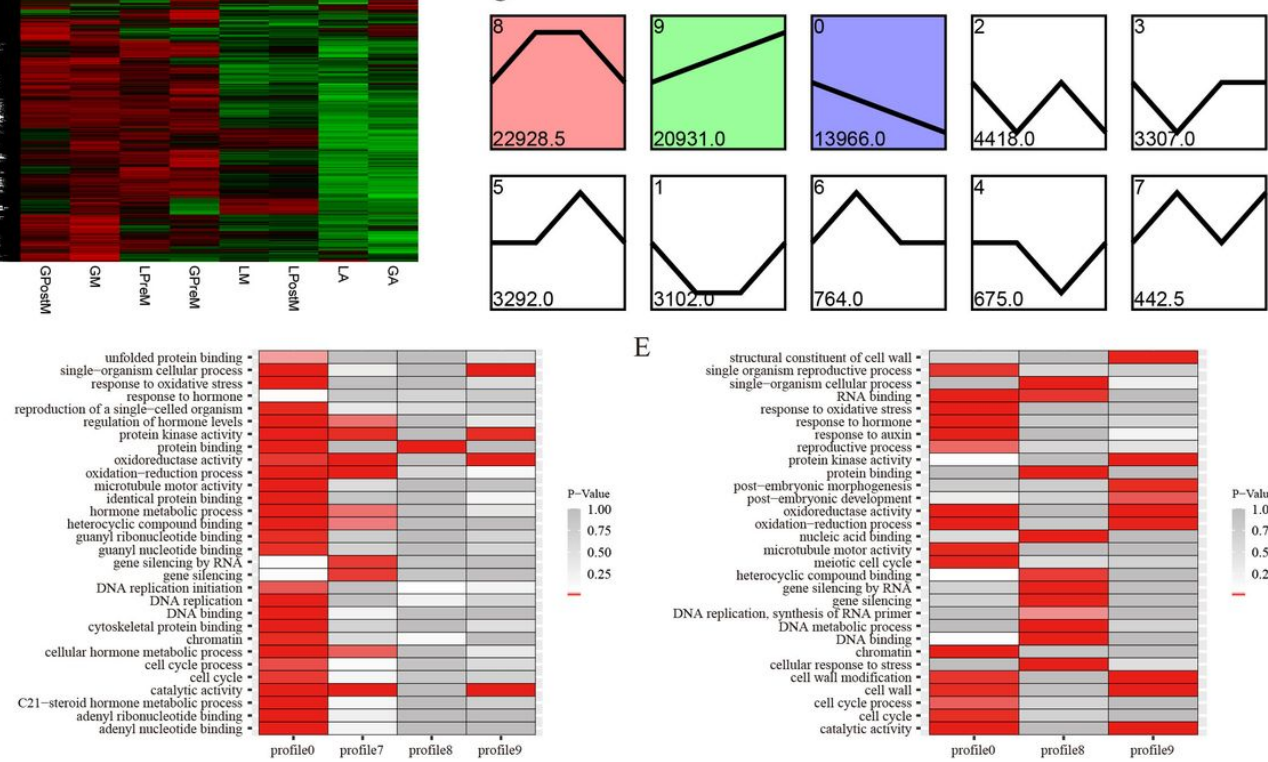

E

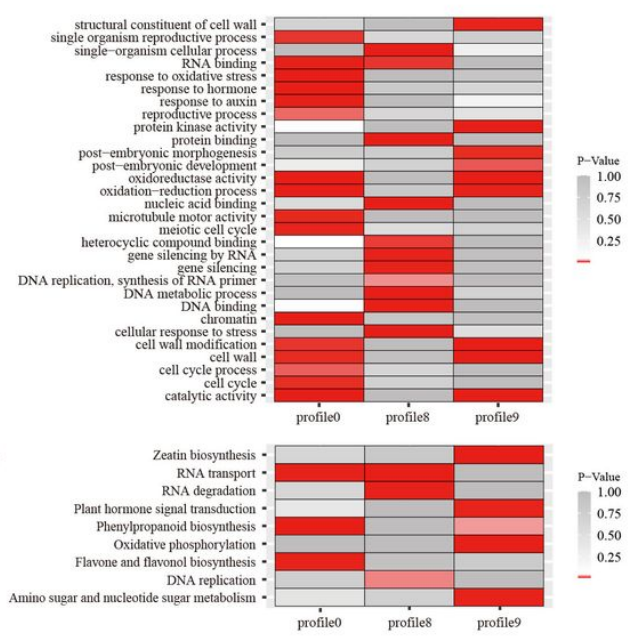

Figure 7

Gene expression patterns and DEGs enrichment of GO terms and KEGG pathways across four time-points in LN and GN. A: Hierarchical clustering analysis of DEG expression based on the log2(fold-change) value. B and C: Gene expression patterns at four time-points in LN and GN. D and E: Results of the GO enrichment analysis of important processes in LN and GN. F and G: Results of the KEGG pathway enrichment analysis of important processes in LN and GN. 
A
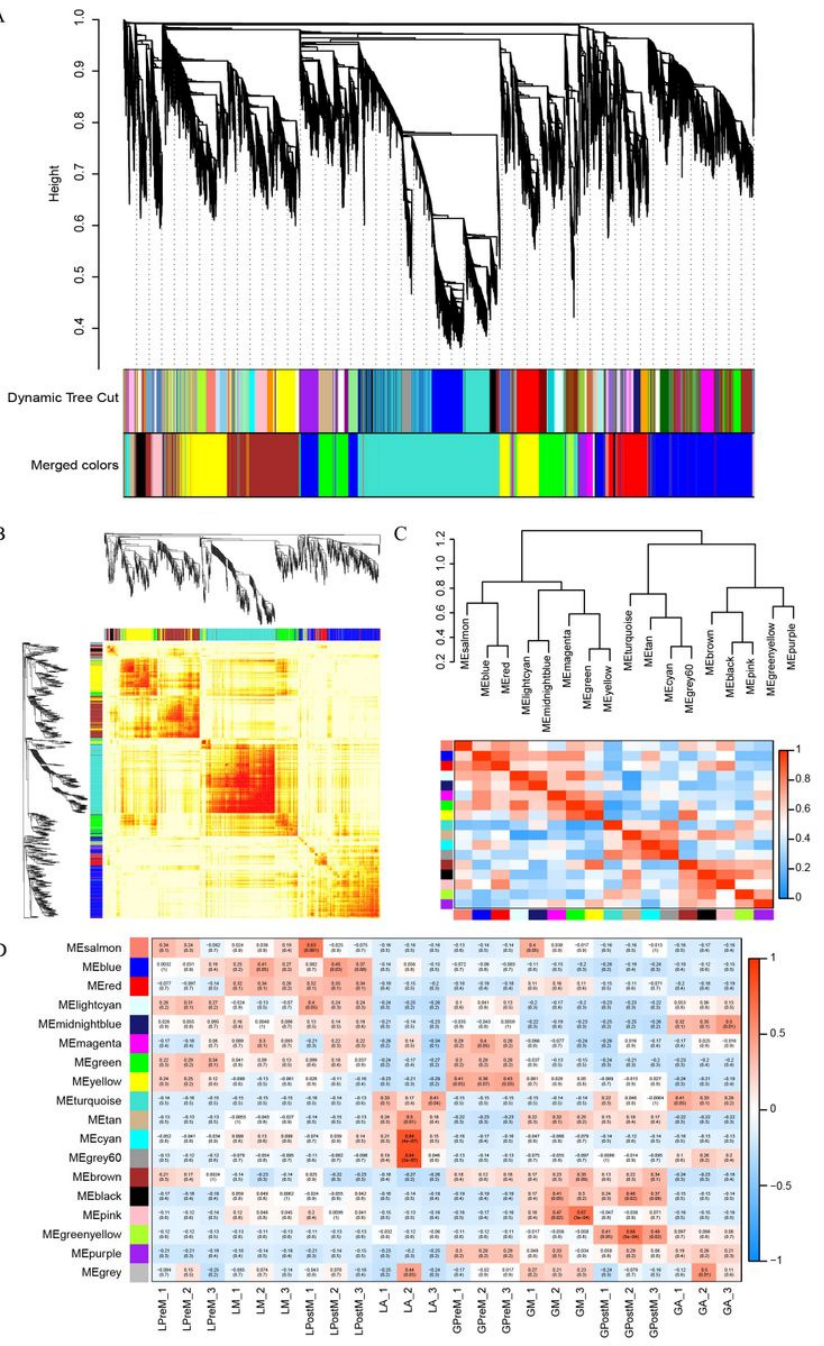

Figure 8

Coexpression network of four stages during embryonic development in Kentucky bluegrass. A: The number of gene hierarchical clusters analyzed by the coexpression network of LN and GN. B: 18 modules of the correlation analysis of the heatmap. C: The relationship between the 18 modules and their correlation. D: Sample and module correlation heatmap. 

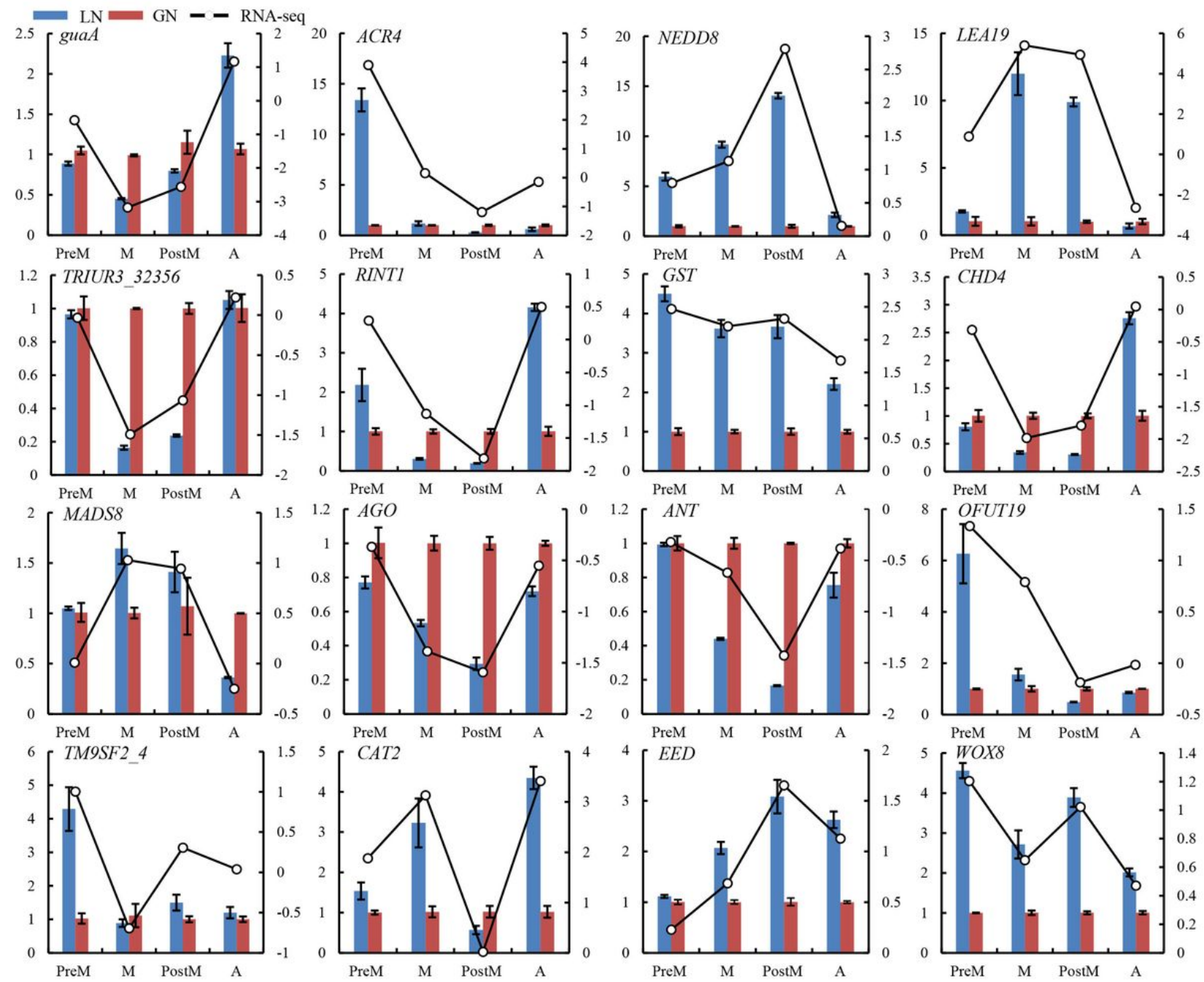

Figure 9

The relative abundance of selected genes was verified by qRT-PCR. Bars with standard errors represent relative expression levels determined by qRT-PCR (left $y$-axis). Broken lines indicate transcript abundance change (log2(fold-change) value) of RNA-Seq (right y-axis). 


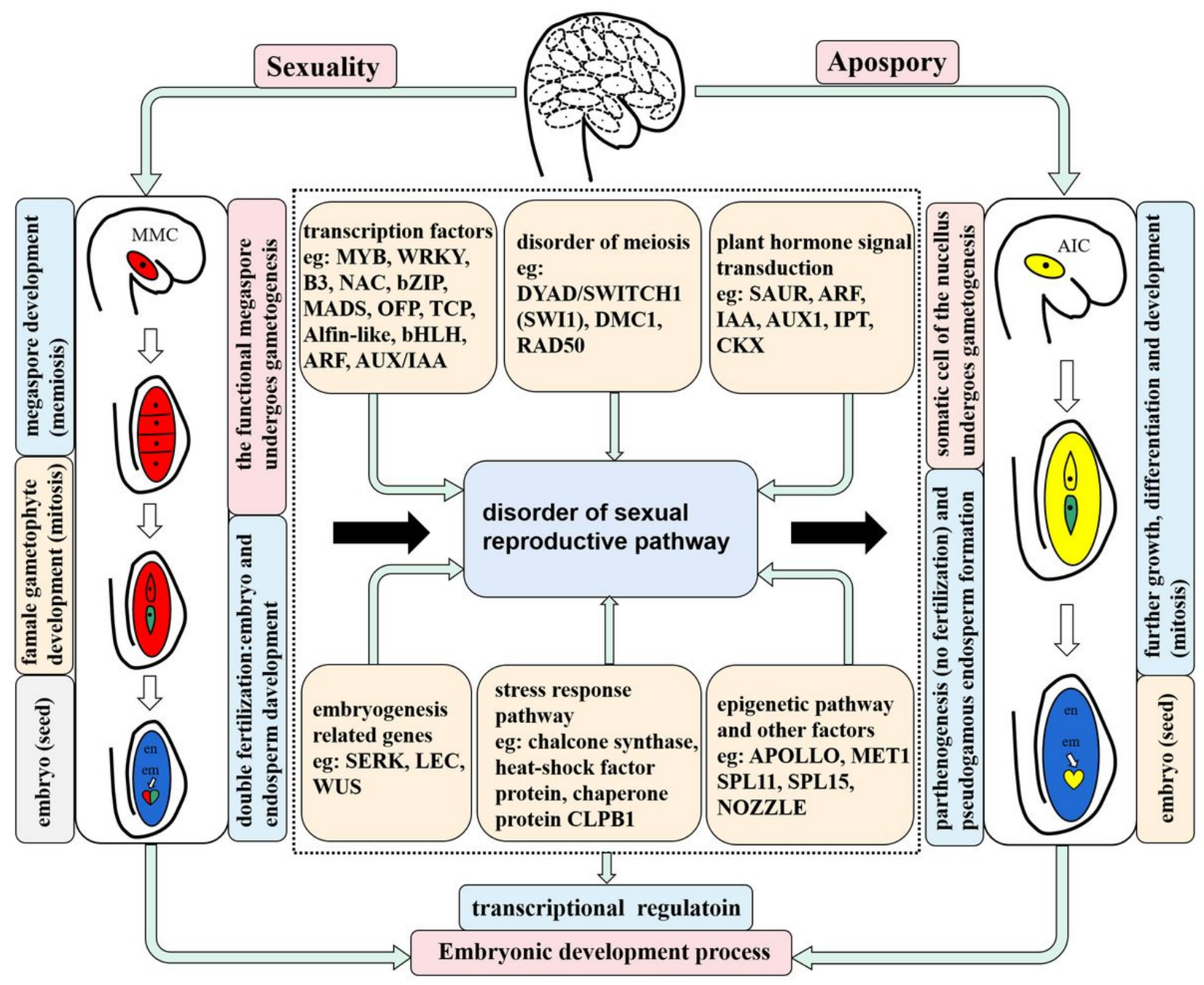

Figure 10

Molecular regulation model of apomixis embryos of Kentucky bluegrass

\section{Supplementary Files}

This is a list of supplementary files associated with this preprint. Click to download.

- SupplementaryFigS1.jpg

- SupplementaryFigS2.jpg

- SupplementaryFigS3.jpg

- SupplementaryTables1.xlsx

- SupplementaryTables2.xlsx

- SupplementaryTableS3.xlsx

- SupplementaryTableS4.xlsx

- SupplementaryTableS5.xlsx

- SupplementaryTableS6.xlsx

- SupplementaryTableS7.xIsx

- SupplementaryTableS8.xlsx

- SupplementaryTableS9.xlsx

- SupplementaryTableS10.xlsx

- SupplementaryTableS11.xlsx

- SupplementaryTableS12.xlsx

- SupplementaryTableS13.xlsx

- SupplementaryTableS14.xlsx 
- SupplementaryTableS15.xlsx

Page 24/24 